\title{
Cup products, the Johnson homomorphism and surface bundles over surfaces with multiple fiberings
}

\author{
NiCK SALTER
}

Let $\Sigma_{g} \rightarrow E \rightarrow \Sigma_{h}$ be a surface bundle over a surface with monodromy representation $\rho: \pi_{1} \Sigma_{h} \rightarrow \operatorname{Mod}\left(\Sigma_{g}\right)$ contained in the Torelli group $\mathcal{I}_{g}$. We express the cup product structure in $H^{*}(E, \mathbb{Z})$ in terms of the Johnson homomorphism $\tau: \mathcal{I}_{g} \rightarrow \bigwedge^{3}\left(H_{1}\left(\Sigma_{g}, \mathbb{Z}\right)\right) / H_{1}\left(\Sigma_{g}, \mathbb{Z}\right)$. This is applied to the question of obtaining an upper bound on the maximal $n$ such that $p_{1}: E \rightarrow \Sigma_{h_{1}}, \ldots, p_{n}: E \rightarrow \Sigma_{h_{n}}$ are fibering maps realizing $E$ as the total space of a surface bundle over a surface in $n$ distinct ways. We prove that any nontrivial surface bundle over a surface with monodromy contained in the Johnson kernel $\mathcal{K}_{g}$ fibers in a unique way.

57R22; 57R95

\section{Introduction}

The theory of the Thurston norm gives a detailed picture of the set of possible ways that a compact, oriented 3-manifold $M$ can fiber as a surface bundle. If $b_{1}(M)>1$, then $M$ admits infinitely many such fibrations $\Sigma_{g} \rightarrow M \rightarrow S^{1}$, finitely many for each $g \geq 2$. The purpose of the present paper is to take up a similar sort of inquiry for 4-manifolds $\Sigma_{g} \rightarrow E \rightarrow \Sigma_{h}$ fibering as a surface bundle over a surface of genus $g \geq 2$.

When $h=1$ (ie the base surface is a torus), a similar story as in the 3-manifold setting unfolds; if $M^{3}$ is a 3-manifold admitting infinitely many fiberings $p: M \rightarrow S^{1}$, then $p \times$ id: $M^{3} \times S^{1} \rightarrow S^{1} \times S^{1}$ admits infinitely many fiberings as well. However, in stark contrast with the 3-dimensional setting and with the case of surface bundles over the torus, FE A Johnson [8] showed that if $\Sigma_{g} \rightarrow E \rightarrow \Sigma_{h}$ is a surface bundle over a surface with $g, h \geq 2$, then there are only finitely many distinct fibrations $p_{i}: E \rightarrow \Sigma_{h_{i}}$ realizing $E$ as the total space of a surface bundle over a surface (see Proposition 2.1 for a precise definition of what is meant by "distinct"). Hillman [7] contains a treatment of results of this type, as does Rivin [12], in which the case of surface bundles over surfaces is situated in the larger context of "fibering rigidity" for a wide class of manifolds.

A particularly simple example of a surface bundle over a surface admitting two fiberings is that of a trivial bundle, ie a product of surfaces $\Sigma_{g} \times \Sigma_{h}$. At the time of 
Johnson's result, there was essentially one known method for producing nontrivial surface bundles over surfaces with multiple fiberings, due independently to Atiyah [1] and Kodaira [9] (see also the summary in [11]). Their construction is built by taking a certain cyclic branched covering $p: E \rightarrow \Sigma_{g} \times \Sigma_{h}$ of a product of surfaces. The two fibering maps are inherited from the projections of $\Sigma_{g} \times \Sigma_{h}$ onto either factor. While Johnson's argument produces a bound on the number of possible fiberings of a surface bundle $E$ that is super-exponential in the Euler characteristic $\chi(E)$, until recently all known examples of surface bundles over surfaces had at most two fiberings, leaving a large gap between the upper and lower bounds on the number of possible fiberings.

The author [14] gave a new method for constructing surface bundles over surfaces with multiple fiberings, including the first examples of bundles admitting an arbitrarily large number of fiberings. In fact, the methods of [14] are capable of producing families $E_{n}$ of surface bundles admitting exponentially many fiberings as a function of $\chi\left(E_{n}\right)$. The results of this paper can be seen as a complement to that work, in that our concern here is in addressing the question of when surface bundles over surfaces admit unique fiberings.

A central theme in the study of surface bundles is the "monodromy-topology dictionary". For any reasonable base space $M$, there is a well-known correspondence (see eg Farb and Margalit [3])

$$
\left\{\begin{array}{l}
\text { bundle isomorphism classes of } \\
\text { oriented } \Sigma_{g} \text {-bundles over } M
\end{array}\right\} \longleftrightarrow\left\{\begin{array}{l}
\text { conjugacy classes of represen- } \\
\text { tations } \pi_{1}(M) \rightarrow \operatorname{Mod}\left(\Sigma_{g}\right)
\end{array}\right\}
$$

This raises the question of translating between topological and geometric properties of surface bundles on the one hand and, on the other, algebraic or geometric properties of the monodromy representation. Certain entries in this dictionary are well established, for instance Thurston's landmark result that a fibered 3-manifold $\Sigma_{g} \rightarrow M_{\phi} \rightarrow S^{1}$ admits a complete hyperbolic metric if and only if the monodromy is a so-called "pseudo-Anosov" element of $\operatorname{Mod}\left(\Sigma_{g}\right)$. In this paper we add to the dictionary by relating the cohomology ring of a surface bundle over a surface to its monodromy representation, then apply these results to give various obstructions for the surface bundle to admit more than one fibering.

From the perspective of the monodromy representation, the phenomenon of multiple fibering remains mysterious. The central result of this paper shows that there is a strong interaction between the existence of multiple fiberings and the theory of the Torelli group $\mathcal{I}_{g}$. Recall that the Torelli group is the kernel of the symplectic representation 
$\Psi: \operatorname{Mod}\left(\Sigma_{g}\right) \rightarrow \operatorname{Sp}_{2 g}(\mathbb{Z})$ and that the Johnson kernel $\mathcal{K}_{g}$ is defined as the group generated by Dehn twists $T_{\gamma}$ with $\gamma$ a separating curve. ${ }^{1}$

Theorem 1.1 Let $\pi: E \rightarrow B$ be a surface bundle over a surface with monodromy in the Johnson kernel $\mathcal{K}_{g}$. If $E$ admits two distinct fiberings then $E$ is diffeomorphic to $B \times B^{\prime}$, the product of the base spaces. In other words, any nontrivial surface bundle over a surface with monodromy in $\mathcal{K}_{g}$ admits a unique fibering.

The surface bundles over surfaces of [14] can be constructed so as to have monodromy contained in $\mathcal{I}_{g}$. It follows that the hypothesis in Theorem 1.1 that the monodromy be contained in $\mathcal{K}_{g}$ is effectively sharp with respect to the Johnson filtration (see [3, Chapter 6] for the definition of the Johnson filtration).

Theorem 1.1 is proved by first relating the monodromy representation of a surface bundle over a surface $E^{4} \rightarrow B^{2}$ to the cohomology ring $H^{*}(E)$. This analysis will show that the integral cohomology of a surface bundle over a surface with monodromy in $\mathcal{K}_{g}$ is as simple as possible. It is then shown that, in these circumstances, obstructions to possessing alternative fiberings can be extracted from $H^{*}(E)$.

In a similar spirit we also have the following general criterion, which we believe to be of independent interest, for a surface bundle over a surface to possess a unique fibering. It can be viewed as the 4-manifold analogue of a well-known fact about fibered 3-manifolds (see Remark 3.6).

Theorem 3.5 Let $p: E \rightarrow B$ be a surface bundle over a surface $B$ of genus $g \geq 2$ with monodromy representation $\rho: \pi_{1} B \rightarrow \operatorname{Mod}\left(\Sigma_{g}\right)$. Suppose that the space of invariant cohomology $\left(H^{1}(F, \mathbb{Q})\right)^{\rho}$ (equivalently, the coinvariant homology of the fiber $\left.\left(H_{1}(F, \mathbb{Q})\right)_{\rho}\right)$ vanishes. Then $E$ admits a unique fibering.

The paper is organized as follows. In Section 2, we give various characterizations of the notion of equivalence under consideration. In Section 3, we prove Theorem 3.5. Sections 4-7 are devoted to the proof of Theorem 1.1. Section 4 is devoted to a lemma in differential topology that features in later stages of the proof of Theorem 1.1. The technical heart of the paper is Section 5. In it, we first give an overview of the classical description of the Johnson homomorphism $\tau$ in terms of the intersection theory of surfaces in 3-manifolds that fiber over $S^{1}$. Using this description of $\tau$, we then carry out a construction of 3-manifolds embedded in surface bundles over surfaces that

\footnotetext{
${ }^{1}$ As discussed further in Section 5.1, there is an alternative characterization of $\mathcal{K}_{g}$ as the kernel of the Johnson homomorphism (to be defined there). We will pass between these two perspectives as the situation dictates.
} 
realizes the relationship between the Johnson homomorphism and the intersection product in the homology of the surface bundle. We give a complete description of the product structure in (co)homology for a surface bundle over a surface with monodromy in $\mathcal{I}_{g}$. These methods of Section 5 extend to an arbitrary surface bundle over a surface, but we do not state them in this level of generality since we have no need for them here.

Section 6 is devoted to some technical results concerning multisections of surface bundles, and their connection to splittings on rational cohomology. These results are used in the course of proving Theorem 1.1.

In Section 7 we turn finally to the proof of Theorem 1.1. The result follows from an analysis of the intersection product structure in $H_{*}(E)$ for a surface bundle over a surface $\Sigma_{g} \rightarrow E \rightarrow \Sigma_{h}$ with monodromy in $\mathcal{K}_{g}$. The results of Section 5 are applied to show that if the monodromy of $\Sigma_{g} \rightarrow E \rightarrow \Sigma_{h}$ is contained in $\mathcal{K}_{g}$, then $E$, which necessarily has $H^{*} E \approx H^{*} \Sigma_{g} \otimes H^{*} \Sigma_{h}$ as an additive group, in fact has $H^{*} E \approx H^{*} \Sigma_{g} \otimes H^{*} \Sigma_{h}$ (with $\mathbb{Z}$ coefficients) as a graded ring. This condition is then exploited to prove Theorem 1.1.

Acknowledgements The author would like to express his gratitude to Tom Church, Sebastian Hensel, Jonathan Hillman, Andy Putman, and Alden Walker for illuminating discussions at various stages of this work. He is grateful to the anonymous referees for many helpful suggestions. He would also like to extend his warmest thanks to Benson Farb for his extensive comments as well as his invaluable support from start to finish.

\section{Equivalence}

If $E$ is a smooth $n$-manifold and $p_{i}: E \rightarrow B_{i}, i=1, \ldots, k$, are projection maps for various fiber bundle structures on $E$, we can consider the product of all the projection maps

$$
p_{1} \times \cdots \times p_{k}: E \rightarrow B_{1} \times \cdots \times B_{k} .
$$

In particular, if $E^{4}$ is the total space of a surface bundle over a surface with two fiberings, the bi-projection $p_{1} \times p_{2}: E \rightarrow B_{2} \times B_{2}$ is defined. As remarked in the introduction, ultimately we are concerned with fiberwise diffeomorphism classes of surface bundles. However, it is convenient to consider a more restrictive notion of equivalence, which will turn out to have the advantage of being describable purely on the level of the fundamental group. 
We say that two fiberings $p_{1}: E \rightarrow B_{1}$ and $p_{2}: E \rightarrow B_{2}$ are $\pi_{1}$-fiberwise diffeomorphic if (1) they are fiberwise diffeomorphic, ie there exists a commutative diagram

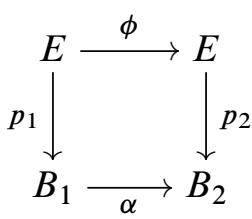

with $\phi, \alpha$ diffeomorphisms, and (2) $\phi_{*}\left(\pi_{1} F_{1}\right)=\pi_{1} F_{1}$ (here, as always, $F_{i}$ denotes a fiber of $\left.p_{i}\right)$. Certainly if $p_{1}$ and $p_{2}$ are $\pi_{1}$-fiberwise diffeomorphic bundle structures, then they are fiberwise diffeomorphic in the usual sense. We are interested in this notion because we want to always regard the trivial bundle $\Sigma_{g} \times \Sigma_{h}$ as having two distinct fiberings. In the setting of fiberwise diffeomorphism, the projections onto either factor of $\Sigma_{g} \times \Sigma_{g}$ yield equivalent fiberings via the factor-swapping map $\phi(x, y)=$ $(y, x)$, which covers the identity on $\Sigma_{g}$, but $\phi_{*}\left(\pi_{1}\left(\Sigma_{g} \times\{p\}\right)\right) \neq \pi_{1}\left(\Sigma_{g} \times\{p\}\right)$. The following proposition asserts that $\pi_{1}$-fiberwise diffeomorphism classes are in correspondence with the fiber subgroups $\pi_{1} F \triangleleft \pi_{1} E$. Recall that this is the setting in which FE A Johnson proved his finiteness result (see [8]).

Proposition 2.1 Suppose $E$ is the total space of a surface bundle over a surface in two ways, $p_{1}: E \rightarrow B_{1}$ and $p_{2}: E \rightarrow B_{2}$. Let $F_{1}$ and $F_{2}$ denote fibers of $p_{1}$ and $p_{2}$, respectively. Then the following are equivalent:

(1) The fiberings $p_{1}$ and $p_{2}$ are $\pi_{1}$-fiberwise diffeomorphic.

(2) The fiber subgroups $\pi_{1} F_{1}, \pi_{1} F_{2} \leq \pi_{1} E$ are equal.

If $\operatorname{deg}\left(p_{1} \times p_{2}\right) \neq 0$ then the bundle structures $p_{1}$ and $p_{2}$ are distinct.

Proof First suppose that $p_{1}$ and $p_{2}$ are equivalent. Appealing to the long exact sequence in homotopy, we see that:

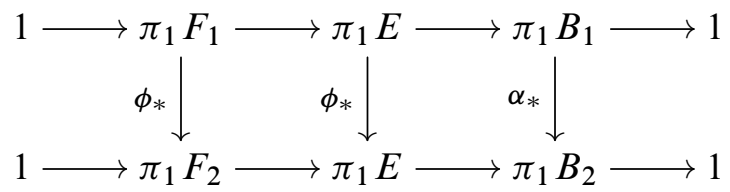

By assumption, $\phi_{*}\left(\pi_{1} F_{1}\right)=\pi_{1} F_{1}$, so that (1) implies (2).

Conversely, suppose that $\pi_{1} F_{1}=\pi_{1} F_{2}$. Then the bundle structures $p_{1}$ and $p_{2}$ give rise to the same splitting

$$
1 \rightarrow \pi_{1} F \rightarrow \pi_{1} E \rightarrow \pi_{1} B \rightarrow 1
$$


on fundamental groups. The monodromy for each bundle can be obtained from this sequence via the map $\pi_{1} B \rightarrow \operatorname{Out}\left(\pi_{1} F\right) \approx \operatorname{Mod}\left(\Sigma_{g}\right)$. This shows that the monodromies for the two bundle structures are conjugate and so, via the correspondence (1), there is a bundle isomorphism $\phi: E \rightarrow E$ covering the identity on $B$. To see that $\phi_{*}\left(\pi_{1} F_{1}\right)=\pi_{1} F_{1}$, consider the induced map on the long exact sequence in homotopy coming from $\phi$ :

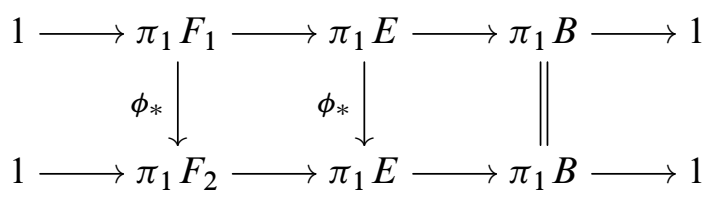

This shows $\phi_{*}\left(\pi_{1} F_{1}\right)=\pi_{1} F_{2}$, and $\pi_{1} F_{1}=\pi_{1} F_{2}$ by assumption, so (2) implies (1). It remains to show that if $\operatorname{deg}\left(p_{1} \times p_{2}\right) \neq 0$ then $p_{1}$ and $p_{2}$ are distinct. We establish the contrapositive. Suppose that $\pi_{1} F_{1}=\pi_{1} F_{2}$. For $i=1,2$, we view $\pi_{1} B_{i}$ as the quotient $\pi_{1} B_{i} \approx \pi_{1} E / \pi_{1} F_{i}$. If $p_{1} \times p_{2}$ is the bi-projection then, in this notation,

$$
\left(p_{1} \times p_{2}\right)_{*}: \pi_{1} E \rightarrow \pi_{1} B_{1} \times \pi_{1} B_{2}
$$

is given by

$$
\left(p_{1} \times p_{2}\right)_{*}(x)=\left(x \pi_{1} F_{1}, x \pi_{1} F_{2}\right)=([x],[x]),
$$

where $[x]=x\left(\bmod \pi_{1} F_{1}\right)=x\left(\bmod \pi_{1} F_{2}\right)$. As $\pi_{1} F_{1}=\pi_{1} F_{2}$, the quotients $\pi_{1} B_{1}$ and $\pi_{1} B_{2}$ are isomorphic, and as they are $K(G, 1)$ spaces, there is a homotopy equivalence

$$
f: B_{1} \rightarrow B_{2}
$$

Let $g$ be the map

$$
g=(f \times \mathrm{id}) \circ\left(p_{1} \times p_{2}\right): E \rightarrow B_{2} \times B_{2} .
$$

By the above,

$$
\operatorname{Im}(g)=\Delta=\left\{(x, x) \mid x \in B_{2}\right\} .
$$

Being nonsurjective, $g$ has degree 0 . As $p_{1} \times p_{2}$ is the composition of $g$ with a homotopy equivalence, we conclude that also $\operatorname{deg}\left(p_{1} \times p_{2}\right)=0$.

In general the condition $\operatorname{deg}\left(p_{1} \times p_{2}\right)=0$ on a bi-projection does not imply that the associated fiberings are equivalent. However, in the setting of the Johnson kernel, this is indeed the case.

Proposition 2.2 Suppose $E$ is the total space of a surface bundle over a surface in two ways, $p_{1}: E \rightarrow B_{1}$ and $p_{2}: E \rightarrow B_{2}$. Let $F_{1}$ and $F_{2}$ denote fibers of $p_{1}$ and $p_{2}$, respectively. Suppose that $\rho_{1}: \pi_{1} B_{1} \rightarrow \operatorname{Mod}\left(F_{1}\right)$ is contained in the Johnson kernel $\mathcal{K}_{g}$. Then the following are equivalent: 
(1) The fiberings $p_{1}$ and $p_{2}$ are not $\pi_{1}$-fiberwise diffeomorphic.

(2) The fiber subgroups $\pi_{1} F_{1}, \pi_{1} F_{2} \leq \pi_{1} E$ are distinct.

(3) $\operatorname{deg}\left(p_{1} \times p_{2}\right) \neq 0$.

(4) $E$ is diffeomorphic to $B_{1} \times B_{2}$.

The additional assertions in Proposition 2.2 will be proved in the course of establishing Theorem 1.1 (see Remark 7.6).

\section{Surface bundles over surfaces with unique fiberings}

In this section, we prove Theorem 3.5. The additive structure of $H^{*} E$ is central to everything that follows in the paper, so we begin with a review of the relevant results. The following theorem was formulated and proved by Morita [10] for the case of field coefficients of characteristic not dividing $\chi(F)$; subsequently this was improved to integral coefficients in the cohomological setting by Cavicchioli, Hegenbarth and Repovš [2].

Proposition 3.1 (Morita, Cavicchioli-Hegenbarth-Repovš) Let $F$ be a closed surface of genus $g \geq 2$. The Serre spectral sequence (with twisted coefficients) of any surface bundle $F \rightarrow E \rightarrow B$ collapses at the $E_{2}$ page. Consequently, there are noncanonical isomorphisms for all $k$,

$$
\begin{aligned}
& H_{k}(E, \mathbb{Q})=H_{k}(B, \mathbb{Q}) \oplus H_{k-1}\left(B, H_{1}(F, \mathbb{Q})\right) \oplus H_{k-2}(B, \mathbb{Q}), \\
& H^{k}(E, \mathbb{Z})=H^{k}(B, \mathbb{Z}) \oplus H^{k-1}\left(B, H^{1}(F, \mathbb{Z})\right) \oplus H^{k-2}(B, \mathbb{Z}) .
\end{aligned}
$$

The $H_{k-2} B$ summand of $H_{k} E$ is canonical and is realized by the Gysin map $p^{!}$, which associates to a homology class $x \in B$ the induced sub-bundle $E_{x}$ sitting over $x$. Similarly, the $H^{k} B$ summand is canonical via the pullback map $p^{*}: H^{k} B \rightarrow H^{k} E$. If $F \rightarrow E \rightarrow B$ has monodromy in $\mathcal{I}_{g}$, then the coefficient system is untwisted and $H^{*}(E, \mathbb{Z}) \approx H^{*}(B, \mathbb{Z}) \otimes H^{*}(F, \mathbb{Z})$ additively. In particular, $H^{*}(E, \mathbb{Z})$ is torsion-free and so, by the universal coefficients theorem, there is also an isomorphism $H_{*}(E, \mathbb{Z}) \approx H_{*}(B, \mathbb{Z}) \otimes H_{*}(F, \mathbb{Z})$.

Because the surface bundles we will be considering in this paper have monodromy lying in $\mathcal{I}_{g}$, we will subsequently take all coefficients to be $\mathbb{Z}$ without further mention. A remark, which is obvious from Proposition 3.1, is that if $*$ generates $H_{0}(B)$ then $p^{!}(*)$ is a primitive class; we will use this fact later on. Here and throughout, we will use the notation

$$
[F]=p^{!}(*) \in H_{2}(E)
$$


to denote the (pushforward of the) fundamental class of the fiber.

The following result is a well-known application of the theory of the Gysin homomorphism and we state it without proof.

Proposition 3.2 Let $p: E \rightarrow B$ be a surface bundle with fiber $F$. If $\chi(F) \neq 0$, then there are injections

$$
\begin{aligned}
p^{*}: H^{*}(B, \mathbb{Q}) & \rightarrow H^{*}(E, \mathbb{Q}), \\
p^{!}: H_{k}(B, \mathbb{Q}) & \rightarrow H_{k+2}(E, \mathbb{Q}) .
\end{aligned}
$$

In the case where $H_{*}(E, \mathbb{Z})$ is torsion-free, the same statements hold with $\mathbb{Z}$ coefficients. In particular, this is true whenever $E$ has monodromy lying in $\mathcal{I}_{g}$, since in this case $H^{*}(E, \mathbb{Z})$ is isomorphic to $H^{*}(F, \mathbb{Z}) \otimes H^{*}(B, \mathbb{Z})$ as an abelian group (see Proposition 3.1).

For surface bundles over surfaces with multiple fiberings, there is an extension of the previous result.

Lemma 3.3 Let $E$ be a 4-manifold with two distinct surface bundle structures $p_{1}: E \rightarrow B_{1}$ and $p_{2}: E \rightarrow B_{2}$. Then

$$
p_{1}^{*}\left(H^{1}\left(B_{1}, \mathbb{Q}\right)\right) \cap p_{2}^{*}\left(H^{1}\left(B_{2}, \mathbb{Q}\right)\right)=\{0\}
$$

and so, by Proposition 3.2, there is a canonical injection

$$
p_{1}^{*} \times p_{2}^{*}: H^{1}\left(B_{1}, \mathbb{Q}\right) \oplus H^{1}\left(B_{2}, \mathbb{Q}\right) \hookrightarrow H^{1}(E, \mathbb{Q}) .
$$

Proof By the universal coefficients theorem, for any space $X$ there is an identification

$$
H^{1}(X, \mathbb{Q}) \approx \operatorname{Hom}\left(\pi_{1} X, \mathbb{Q}\right) .
$$

Under this identification, a character $\alpha \in \operatorname{Hom}\left(\pi_{1} B_{i}, \mathbb{Q}\right)$ is pulled back to $p_{i}^{*}(\alpha)$ in $\operatorname{Hom}\left(\pi_{1} E, \mathbb{Q}\right)$ by precomposition with $\left(p_{i}\right)_{*}$. In particular, $p_{i}^{*}(\alpha)$ vanishes on $\pi_{1} F_{i}=\operatorname{ker}\left(p_{i}\right)_{*}$. Therefore, any character $\alpha \in p_{1}^{*}\left(H^{1}\left(B_{1}, \mathbb{Q}\right)\right) \cap p_{2}^{*}\left(H^{1}\left(B_{2}, \mathbb{Q}\right)\right)$ must vanish on the subgroup generated by $\left(\pi_{1} F_{1}\right)\left(\pi_{1} F_{2}\right)$.

By Lemma 3.4 below, $\left(\pi_{1} F_{1}\right)\left(\pi_{1} F_{2}\right)$ has finite index in $\pi_{1} E$. For any group $\Gamma$, any character $\alpha: \Gamma \rightarrow \mathbb{Q}$ vanishing on a finite-index subgroup must vanish identically, proving the claim.

Lemma 3.4 Let $E$ be a surface bundle over a surface with two distinct fiberings $p_{i}: E \rightarrow B_{i}, i=1,2$; let the fibers be $F_{1}$ and $F_{2}$, respectively. Then $\left(\pi_{1} F_{1}\right)\left(\pi_{1} F_{2}\right)$ has finite index in $\pi_{1} E$. 
Proof Consider the cross-projection $\pi_{1} F_{1} \rightarrow \pi_{1} B_{2}$. Let the image of $\pi_{1} F_{1}$ in $\pi_{1} B_{2}$ be denoted by $\Gamma$. This is a finitely generated normal subgroup of $\pi_{1} B_{2}$. For any surface group of genus $g \geq 2$, any nontrivial finitely generated normal subgroup has finite index (see [8, Property (D6)]). If $\Gamma$ is the trivial group, then $\pi_{1} F_{1} \leq \pi_{1} F_{2}$, necessarily again of finite index. In this case, the image of $\pi_{1} F_{2}$ in $\pi_{1} B_{1}$ is therefore finite, but $\pi_{1} B_{1}$ is torsion-free. We conclude that $\Gamma \leq \pi_{1} B_{2}$ has finite index. The kernel of the map $\pi_{1} E \rightarrow\left(\pi_{1} B_{2} / \Gamma\right)$ is exactly $\left(\pi_{1} F_{1}\right)\left(\pi_{1} F_{2}\right)$.

Recall that if $\rho: G \rightarrow \operatorname{GL}(V)$ is a representation then the invariant space $V^{\rho}$ is defined by

$$
V^{\rho}=\{v \in V \mid \rho(g)(v)=v \text { for all } g \in G\} .
$$

The space of coinvariants $V_{\rho}$ of the representation is defined as

$$
V_{\rho}=V / W, \quad \text { where } W=\{v-\rho(g)(v) \mid v \in V, g \in G\} .
$$

Theorem 3.5 Let $p: E \rightarrow B$ be a surface bundle over a surface $B$ of genus $g \geq 2$ with monodromy representation $\rho: \pi_{1} B \rightarrow \operatorname{Mod}\left(\Sigma_{g}\right)$. Suppose that the space of invariant cohomology $\left(H^{1}(F, \mathbb{Q})\right)^{\rho}$ (equivalently, the coinvariant homology of the fiber $\left.\left(H_{1}(F, \mathbb{Q})\right)_{\rho}\right)$ vanishes. Then $E$ admits a unique fibering.

Proof For any surface bundle $p: E \rightarrow B$ with monodromy $\rho$ and any choice of coefficients, there is a (noncanonical) splitting

$$
H^{1}(E)=p^{*}\left(H^{1}(B)\right) \oplus\left(H^{1}(F)\right)^{\rho}
$$

(see Proposition 3.1). If $\left(H^{1}(F, \mathbb{Q})\right)^{\rho}=0$, then this reduces to

$$
H^{1}(E, \mathbb{Q})=p^{*} H^{1}(B, \mathbb{Q}) .
$$

If $p_{2}: E \rightarrow B_{2}$ is a second, distinct fibering, the above shows that

$$
p_{2}^{*}\left(H^{1}\left(B_{2}, \mathbb{Q}\right)\right) \leq p^{*} H^{1}(B, \mathbb{Q}) .
$$

However, this contradicts Lemma 3.3.

Remark 3.6 Recall that a surface bundle over $S^{1}$, viewed as the mapping torus $M$ of some diffeomorphism $\phi$ of a surface $F$, admits a unique fibering if and only if $b_{1}(M)=1$. This is the case exactly when $\left(H_{1}(F, \mathbb{Q})\right)_{\phi}=0$, so Theorem 3.5 is the counterpart to this fact in dimension 4. Moreover, a random element $\phi \in \operatorname{Mod}\left(\Sigma_{g}\right)$ satisfies $\left(H_{1}(F, \mathbb{Q})\right)_{\phi}=0$ (see [13]). It easily follows that a generic monodromy representation will also have $\left(H_{1}(F, \mathbb{Q})\right)_{\rho}=0$ : "most" surface bundles over surfaces have a single fibering. The proof of Theorem 3.5 is special to the case of surface bundles over surfaces and it is not clear if Theorem 3.5 is true in greater generality. 


\section{Bi-projections}

In this section we state and prove the key lemma from differential topology needed for the proof of Theorem 1.1.

Proposition 4.1 Let $E$ be a 4-manifold with surface bundle structures $p_{1}$ : $E \rightarrow B_{1}$ and $p_{2}: E \rightarrow B_{2}$. Let $F_{1}$ and $F_{2}$ denote fibers of $p_{1}$ and $p_{2}$ lying over a regular value of $p_{1} \times p_{2}$. If $\operatorname{deg}\left(p_{1} \times p_{2}: E \rightarrow B_{1} \times B_{2}\right) \neq 0$, then the following five quantities are equal:

(1) $\operatorname{deg}\left(p_{1} \times p_{2}: E \rightarrow B_{1} \times B_{2}\right)$.

(2) $\operatorname{deg}\left(\left.p_{1}\right|_{F_{2}}: F_{2} \rightarrow B_{1}\right)$.

(3) $\operatorname{deg}\left(\left.p_{2}\right|_{F_{1}}: F_{1} \rightarrow B_{2}\right)$.

(4) The algebraic intersection number $I_{E}\left(F_{1}, F_{2}\right)$.

(5) The cardinality of the intersection $\left|F \cap F_{2}\right|$.

As (5) indicates, this quantity is always positive.

Proof As $p_{1}$ and $p_{2}$ are projection maps for fiber bundle structures on $E$, they are everywhere regular, and $\operatorname{ker}\left(d p_{1}\right)_{x}$ is identified with the tangent space to the fiber of $p_{1}$ through $x$. Let $z=\left(b_{1}, b_{2}\right) \in B_{1} \times B_{2}$ be a regular value for $p_{1} \times p_{2}$. It follows from the assumption that $\operatorname{deg}\left(p_{1} \times p_{2}: E \rightarrow B_{1} \times B_{2}\right) \neq 0$ that $d\left(p_{1} \times p_{2}\right)_{x}$ is an isomorphism for all $x \in\left(p_{1} \times p_{2}\right)^{-1}(z)$ (and that this preimage is nonempty). The kernel of $d\left(p_{1} \times p_{2}\right)_{x}$ is just the intersection of the kernels of $d\left(p_{1}\right)_{x}$ and $d\left(p_{2}\right)_{x}$. It follows that, for all $x \in\left(p_{1} \times p_{2}\right)^{-1}(z)$,

$$
T_{x} E \approx T_{x} F_{1} \oplus T_{x} F_{2}
$$

Note that this shows that the fibers $F_{1}$ and $F_{2}$ over $b_{1}$ and $b_{2}$, respectively, are transverse.

If orientations on $E, B_{1}$ and $B_{2}$ are chosen properly, then this specifies an orientation on each fiber of $p_{1}$ and $p_{2}$ via the following decomposition, where $H_{x}$ is any complement to $T_{x} F_{1}=\operatorname{ker} d\left(p_{1}\right)_{x}$ :

$$
T_{x} F_{1} \oplus H_{x} \approx T_{x} E .
$$

The orientation on $H_{x}$ is specified by the isomorphism $H_{x} \approx T_{p_{1}(x)} B_{1}$. Of course an analogous convention orients each fiber of $p_{2}$. In particular, it follows from Equation (2) that at any regular point for $p_{1} \times p_{2}$ we can take $H_{x}=T_{x} F_{2}$ and that the restriction of $d\left(p_{1}\right)_{x}$ to $T_{x} F_{2}$ is an isomorphism. 
Recall that if $f: X^{n} \rightarrow Y^{n}$ is a smooth map of oriented closed $n$-manifolds then

$$
\operatorname{deg}(f)=\sum_{x \in f^{-1}(y)} \varepsilon(x)
$$

where $y$ is any regular value of $f$, and $\varepsilon(x)=1$ if the orientation on $T_{y} Y$ induced by $d f_{x}$ agrees with the pre-chosen orientation on $Y$ and $\varepsilon(x)=-1$ otherwise. If $Y$ and $Z$ are smoothly embedded and transversely intersecting oriented submanifolds of the oriented manifold $X$ such that $\operatorname{dim}(X)=\operatorname{dim}(Y)+\operatorname{dim}(Z)$, then the algebraic intersection number of $Y$ and $Z$ is computed as

$$
I_{X}(Y, Z)=\sum_{w \in Y \cap Z} \varepsilon(w)
$$

where $\varepsilon(w)=1$ if the orientation on $T_{w} X$ given by $T_{w} Y \oplus T_{w} Z$ agrees with the pre-chosen orientation on $X$ and $\varepsilon(w)=-1$ otherwise.

It follows from the definitions that

$$
\left(p_{1} \times p_{2}\right)^{-1}\left(b_{1}, b_{2}\right)=\left.p_{1}\right|_{F_{2}} ^{-1}\left(b_{1}\right)=\left.p_{2}\right|_{F_{1}} ^{-1}\left(b_{2}\right)=F_{1} \cap F_{2} .
$$

Therefore, each of the sums computing (1)-(5) take place over the same set of points. So it remains only to show that, in each of the contexts (1)-(4), the relevant orientation convention assigns a positive value.

The orientation number assigned to $x \in\left(p_{1} \times p_{2}\right)^{-1}\left(b_{1}, b_{2}\right)$ is given by the sign of the determinant of the map

$$
d\left(p_{1} \times p_{2}\right)_{x}: T_{x} E \rightarrow T_{b_{1}} B_{1} \oplus T_{b_{2}} B_{2} .
$$

By the above discussion, our orientation convention stipulates that

$$
d\left(\left.p_{1}\right|_{F_{2}}\right)_{x}: T_{x} F_{2} \rightarrow T_{b_{1}} B_{1}
$$

is an orientation-preserving isomorphism and similarly for $d\left(\left.p_{2}\right|_{F_{1}}\right)$. This proves the equality of (2) and (3) with (5).

As

$$
T_{x} F_{1}=\operatorname{ker} d\left(p_{1}\right)_{x} \quad \text { and } \quad T_{x} F_{2}=\operatorname{ker} d\left(p_{2}\right)_{x},
$$

it follows that $d\left(p_{1} \times p_{2}\right)_{x}$ has a block-diagonal decomposition

$$
d\left(p_{1} \times p_{2}\right)_{x}=d\left(p_{1}\right)_{x} \oplus d\left(p_{2}\right)_{x}: T_{x} F_{1} \oplus T_{x} F_{2} \rightarrow T_{b_{2}} B_{2} \oplus T_{b_{1}} B_{1},
$$

from which it follows that $x$ also carries a positive orientation number in setting (1). Finally, the orientation number for $x$ as a point of intersection between $F_{1}$ and $F_{2}$ 
records whether the orientations of $T_{x} E$ and $T_{x} F_{1} \oplus T_{x} F_{2}$ agree, but we have already seen that they necessarily do.

\section{Cup products and the Johnson homomorphism}

The goal of this section is to give a construction of embedded submanifolds in a surface bundle over a surface $E$ that will be explicit enough to compute the intersection form on homology or, dually, the cup product structure in cohomology. One of the original definitions of the Johnson homomorphism was via the cup product structure in surface bundles over $S^{1}$. In this section we turn this perspective on its head and explain how the Johnson homomorphism computes the cup product structure in a surface bundle over a surface (in fact, these methods extend to surface bundles over arbitrary manifolds). The submanifolds we construct will be codimension-1 (ie 3-manifolds) and built so that their intersection theory is explicitly connected to the Johnson homomorphism.

To this end, in Section 5.1 we give a discussion of the definition of the Johnson homomorphism in the setting of the cup product in surface bundles over $S^{1}$. The centerpiece of this is the construction of geometric representatives for classes in $H^{1}$, via embedded surfaces which we call "tube-and-cap surfaces". Then, in Section 5.2, we return to the original problem of constructing representatives for classes in $H^{1}$ of a surface bundle over a surface as embedded 3-manifolds. The construction is carried out so that the intersection of particular pairs of these 3-manifolds is a tube-and-cap surface, thereby realizing the link between cup products in surface bundles over surfaces and the Johnson homomorphism.

\subsection{From the intersection form to the Johnson homomorphism, and back again}

In this subsection we will begin to dive into the theory of the Torelli group in earnest, so we begin with a brief review of the relevant definitions. The Torelli group $\mathcal{I}_{g}$ is the kernel of the symplectic representation $\Psi: \operatorname{Mod}\left(\Sigma_{g}\right) \rightarrow \operatorname{Sp}_{2 g}(\mathbb{Z})$. The Johnson kernel $\mathcal{K}_{g}$ is the subgroup of $\mathcal{I}_{g}$ generated by all Dehn twists $T_{\gamma}$ about separating curves $\gamma$. It is a deep theorem of D Johnson that $\mathcal{K}_{g}$ can alternately be characterized as the kernel of the Johnson homomorphism $\tau$ to be defined below.

Let $\phi \in \mathcal{I}_{g}$ be a Torelli mapping class and build the mapping torus

$$
M_{\phi}=\Sigma_{g} \times I /\{(x, 1) \sim(\phi(x), 0)\} .
$$

As $\phi \in \mathcal{I}_{g}$ for any curve $\gamma \subset \Sigma_{g}$, the homology class $[\gamma]-\phi_{*}[\gamma]$ is zero. Thus there exists a map of a surface $i: S \rightarrow \Sigma_{g}$ which cobounds $\gamma \cup \phi(\gamma)$. Indeed, there exists 
an embedded surface $S \leq \Sigma_{g} \times I$ whose boundary is given by

$$
\partial S=\gamma \times\{1\} \cup \phi(\gamma) \times\{0\} .
$$

To see this, recall that since $S^{1}$ is a $K(\mathbb{Z}, 1)$ there is a correspondence

$$
H^{1}\left(\Sigma_{g}, \mathbb{Z}\right) \approx\left[\Sigma_{g}, S^{1}\right]
$$

Via Poincaré duality,

$$
H^{1}\left(\Sigma_{g}, \mathbb{Z}\right) \approx H_{1}\left(\Sigma_{g}, \mathbb{Z}\right)
$$

The induced correspondence

$$
H_{1}\left(\Sigma_{g}, \mathbb{Z}\right) \approx\left[\Sigma_{g}, S^{1}\right]
$$

is realized by taking the preimage of a regular value, which will be an embedded submanifold. Under this correspondence, homotopic maps $f, g: \Sigma_{g} \rightarrow S^{1}$ yield homologous submanifolds, and conversely. Therefore, the maps $f, g: \Sigma_{g} \rightarrow S^{1}$ which determine $\gamma$ and $\phi(\gamma)$ are homotopic. This gives the desired map $F: \Sigma_{g} \times I \rightarrow S^{1}$ such that the preimage of a regular value is an embedded surface $S$ cobounding $\gamma$ and $\phi(\gamma)$.

In fact, the choice of $S$ is not unique. Let $i^{\prime}: S^{\prime} \rightarrow M_{\phi}$ be any map of a closed surface to $M_{\phi}$. Then the chain $S+S^{\prime}$ satisfies $\partial\left(S+S^{\prime}\right)=\partial S=\gamma-\phi(\gamma)$. Nonetheless, given any $S$ satisfying $\partial(S)=\gamma-\phi(\gamma)$, we can form a closed submanifold of $M_{\phi}$ in the following way. We begin with a tube, diffeomorphic to $S^{1} \times I$, embedded into $M_{\phi}$ as $\phi(\gamma) \times\left[0, \frac{1}{3}\right] \cup \gamma \times\left[\frac{2}{3}, 1\right]$. We may then glue in $S$ to $\Sigma_{g} \times\left[\frac{1}{3}, \frac{2}{3}\right]$. The result is a smoothly embedded oriented submanifold $\Sigma_{\gamma} \subset M_{\phi}$, which will descend to a homology class $\Sigma_{z}$ (here $z=[\gamma]$ ). See Figure 1 .

For convenience, we introduce the following terminology for these surfaces, which we will refer to as tube surfaces. The tube of a tube surface is the cylinder $S^{1} \times I=$ $\phi(\gamma) \times\left[0, \frac{1}{3}\right] \cup \gamma \times\left[\frac{2}{3}, 1\right]$ and the cap is the subsurface $S$.

We assign an orientation to $\Sigma_{\gamma}$ as follows. The tangent space to a point $x$ contained in the tube has a direct sum decomposition

$$
T_{x} \Sigma_{\gamma}=V \oplus T_{x} \gamma
$$

where $V$ is any preimage of $T_{\pi(x)} S^{1}$ and $T_{x} \gamma$ is interpreted as the tangent space to the copy of $\gamma$ sitting in the fiber containing $x$. Both of the summands in (3) have orientations induced from those on $S^{1}$ and $\gamma$, respectively, and this endows $T_{x} \Sigma$ with an orientation. This can then be extended over the cap surface in a coherent way, since $S$ was chosen to be a boundary for $[\gamma]-[\phi(\gamma)]$ with $\mathbb{Z}$ coefficients. 


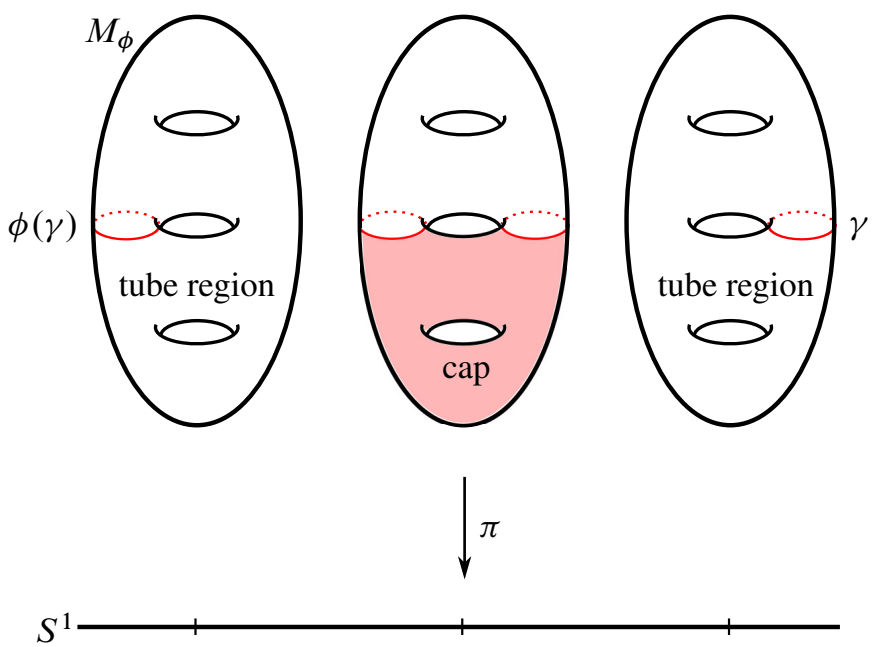

Figure 1: A tube surface

Recall however that the choice of $S$ was not unique. Any closed surface mapping into $\Sigma_{g}$ is homologous to some multiple of the fundamental class, so the above procedure really defines a homomorphism $H_{1}\left(\Sigma_{g}\right) \rightarrow H_{2}\left(M_{\phi}\right) /[F]$, where $[F]$ is the fundamental class of the fiber. If the bundle has a section $\sigma: S^{1} \rightarrow M_{\phi}$, then we can choose $S$ so that $\operatorname{Im} \sigma$ and $\Sigma_{z}$ have zero algebraic intersection, which gives a canonical lift $H_{1}\left(\Sigma_{g}\right) \rightarrow H_{2}\left(M_{\phi}\right)$. In the absence of such auxiliary data, we instead just choose an arbitrary lift and we will account for the consequences later.

Having chosen an embedding $i: H_{1}\left(\Sigma_{g}\right) \hookrightarrow H_{2}\left(M_{\phi}\right)$ such that $z \mapsto \Sigma_{z}$, there is an associated direct sum decomposition of $H_{2}\left(M_{\phi}\right)$, namely

$$
H_{2}\left(M_{\phi}\right)=\langle[F]\rangle \oplus \operatorname{Im} i .
$$

Relative to such an embedding, we form the map $\tau(\phi) \in \operatorname{Hom}\left(\bigwedge^{3} H_{1}\left(\Sigma_{g}\right), \mathbb{Z}\right)$ by

$$
\tau(\phi)(x \wedge y \wedge z)=\Sigma_{x} \cdot \Sigma_{y} \cdot \Sigma_{z},
$$

the term on the right being interpreted as the triple algebraic intersection of the given homology classes. Suppose a section exists and that the $\Sigma_{x}$ have been constructed accordingly. In this case, D Johnson showed that the map

$$
\begin{aligned}
\tau: \mathcal{I}_{g, *} & \rightarrow \operatorname{Hom}\left(\bigwedge^{3} H_{1}\left(\Sigma_{g}\right), \mathbb{Z}\right), \\
\phi & \mapsto \tau(\phi),
\end{aligned}
$$

is a surjective homomorphism. See [3, Chapter 6] for a summary of the Johnson homomorphism, including two alternative definitions. The (pointed) Johnson kernel $\mathcal{K}_{g \text {,* }}$ 
is defined, analogously to the case of closed surfaces, as the subgroup of $\operatorname{Mod}\left(\Sigma_{g, *}\right)$ generated by Dehn twists about separating simple closed curves (scc). As in the closed case, D Johnson established that $\mathcal{K}_{g, *}$ coincides with the kernel of $\tau$. In our context this precisely means that all triple intersections between the various $\Sigma_{x}$ vanish.

Having fixed a family of $\Sigma_{x}$, it is then easy to compute the entire intersection form on $\bigwedge^{3} H_{2}\left(M_{\phi}\right)$. Certainly $[F]^{2}=0$. It is also fairly easy to see that

$$
[F] \cdot \Sigma_{x} \cdot \Sigma_{y}=i(x, y),
$$

where $i(x, y)$ denotes the algebraic intersection pairing in $H_{1}\left(\Sigma_{g}\right)$. Indeed, by picking the choice of fiber to intersect $\Sigma_{x}$ on the tube, it is clear that the result is simply the curve $x$, so that $[F] \cdot \Sigma_{x} \cdot \Sigma_{y}$ computes the intersection of $x$ and $y$ on $F$, at least up to a sign that may be introduced by the (non)compatibilities of the various orientation conventions in play. A quick check reveals this sign to be positive.

We will now be able to account for the ambiguity introduced by our choice of embedding $i: H_{1}\left(\Sigma_{g}\right) \hookrightarrow H_{2}\left(M_{\phi}\right)$, which will in turn lead to the definition of the Johnson homomorphism on the closed Torelli group $\mathcal{I}_{g}$. Suppose that $\Sigma_{w}^{\prime}=\Sigma_{w}+k_{w}[F]$ is some other set of choices that is coherent in the sense that $\Sigma_{w}^{\prime}+\Sigma_{z}^{\prime}=\Sigma_{w+z}^{\prime}$ (ie $x \mapsto k_{x} \in H^{1}\left(\Sigma_{g}\right)$ ). By linearity,

$$
\begin{aligned}
\Sigma_{x}^{\prime} \cdot \Sigma_{y}^{\prime} \cdot \Sigma_{z}^{\prime} & =\Sigma_{x} \cdot \Sigma_{y} \cdot \Sigma_{z}+k_{x} i(y, z)+k_{y} i(z, x)+k_{z} i(x, y) \\
& =\tau(\phi)(x \wedge y \wedge z)+k_{x} i(y, z)+k_{y} i(z, x)+k_{z} i(x, y) \\
& =\tau(\phi)(x \wedge y \wedge z)+C^{*}(k) ;
\end{aligned}
$$

here $C: \bigwedge^{3} H_{1}\left(\Sigma_{g}\right) \rightarrow H_{1}\left(\Sigma_{g}\right)$ is the contraction with the symplectic form $i(\cdot, \cdot)$ and $k \in \operatorname{Hom}\left(H_{1}\left(\Sigma_{g}\right), \mathbb{Z}\right)$ is the form such that $k(w)=k_{w}$. The upshot of this calculation is that $\tau(\phi)$ is well defined as an element of $\operatorname{Hom}\left(\bigwedge^{3} H_{1}\left(\Sigma_{g}\right), \mathbb{Z}\right) / \operatorname{Im} C^{*}$, which can be identified with the more familiar space $\wedge^{3} H / H$ (here we adopt the usual convention that $H=H_{1}\left(\Sigma_{g}\right)$ ). The Johnson homomorphism on the closed Torelli group is then given by

$$
\begin{aligned}
\tau: \mathcal{I}_{g} & \rightarrow \operatorname{Hom}\left(\bigwedge^{3} H_{1}\left(\Sigma_{g}\right), \mathbb{Z}\right) / \operatorname{Im} C^{*} \approx \bigwedge^{3} H / H, \\
\phi & \mapsto \tau(\phi) .
\end{aligned}
$$

As mentioned above, work of D Johnson shows that the kernel of $\tau$ coincides with the previously defined subgroup

$$
\left.\mathcal{K}_{g}=\left\langle T_{\gamma}\right| \gamma \text { separating scc }\right\rangle
$$

Remark 5.1 The construction given above with the tube-and-cap surfaces is a concrete realization of the isomorphism $H_{1}\left(\Sigma_{g}\right) \approx H_{2}\left(M_{\phi}\right) /[F]$ coming from the Serre spectral 
sequence for $p: M_{\phi} \rightarrow S^{1}$. In fact, this same construction will work for an arbitrary $\phi \in \operatorname{Mod}\left(\Sigma_{g}\right)$, yielding an isomorphism $\left(H_{1}\left(\Sigma_{g}\right)\right)^{\phi} \approx H_{2}\left(M_{\phi}\right) /[F]$, but we do not pursue this here.

The above discussion shows how to construct the Johnson homomorphism in terms of the intersection form on $M_{\phi}$. Conversely, we will show next how to reconstruct the intersection form on $M_{\phi}$ from the data of the Johnson homomorphism $\tau(\phi) \in \bigwedge^{3} H / H \approx \operatorname{Hom}\left(\bigwedge^{3} H \Sigma_{g}, \mathbb{Z}\right) / \operatorname{Im} C^{*}$. Begin by selecting an arbitrary lift $\tilde{\tau}(\phi)$ of $\tau(\phi)$ (of course, the presence of a section gives a canonical such choice). Next, construct a coherent family of homology classes $\Sigma_{x}^{\prime}$ by making choices arbitrarily. Define $\tau^{\prime}(\phi) \in \operatorname{Hom}\left(\bigwedge^{3} H, \mathbb{Z}\right)$ by

$$
\tau^{\prime}(\phi)(x \wedge y \wedge z)=\Sigma_{x}^{\prime} \cdot \Sigma_{y}^{\prime} \cdot \Sigma_{z}^{\prime} .
$$

There is no reason to suspect that $\tau^{\prime}(\phi)=\tilde{\tau}(\phi)$. However, as we saw above, we do know that $\tau^{\prime}(\phi)-\tilde{\tau}(\phi) \in \operatorname{Im} C^{*}$, so there is some functional $\alpha \in H^{1}\left(\Sigma_{g}\right)$ such that $\tau^{\prime}(\phi)-\tilde{\tau}(\phi)=C^{*}(\alpha)$. This functional $\alpha$ will allow us to choose the correct set of $\Sigma_{x}$ so that the triple intersections are computed by our choice of $\tilde{\tau}(\phi)$.

Lemma 5.2 We assume the notation of the above setting. By taking

$$
\Sigma_{x}=\Sigma_{x}^{\prime}-\alpha(x)[F]
$$

there is an equality for all $x, y$ and $z$,

$$
\Sigma_{x} \cdot \Sigma_{y} \cdot \Sigma_{z}=\tilde{\tau}(\phi)(x \wedge y \wedge z) .
$$

Proof We compute:

$$
\begin{aligned}
\Sigma_{x} \cdot \Sigma_{y} \cdot \Sigma_{z} & =\Sigma_{x}^{\prime} \cdot \Sigma_{y}^{\prime} \cdot \Sigma_{z}^{\prime}-\alpha(x) i(y, z)-\alpha(y) i(z, x)-\alpha(z) i(x, y) \\
& =\tau^{\prime}(\phi)(x \wedge y \wedge z)-C^{*}(\alpha)(x \wedge y \wedge z) \\
& =\tilde{\tau}(\phi) .
\end{aligned}
$$

\subsection{Intersections in surface bundles over surfaces, and beyond}

The methods of the previous subsection can be adapted to give a description of certain cup products in $H^{1}(E)$, where $p: E^{n+2} \rightarrow B^{n}$ has monodromy lying in $\mathcal{I}_{g}$. The idea will be to define an embedding, as before,

$$
i: H_{1}\left(\Sigma_{g}\right) \hookrightarrow H_{n+1}(E),
$$

by constructing submanifolds $M_{\gamma}$ for curves $\gamma \subset \Sigma_{g}$ by means of a higher-dimensional "tubing construction". Then the triple intersections of collections of $\mathcal{M}_{\gamma}$ will be 
partially computable via the Johnson homomorphism in a certain sense, to be described below. In this subsection we will first briefly sketch the properties we require of the submanifolds $M_{\gamma}$, then we will give the construction. Then, in Section 5.3, we will determine much of the intersection pairing in $H_{*}(E, \mathbb{Z})$.

Our construction will provide, for each simple closed curve $\gamma \subset F$, a submanifold $M_{\gamma}$ such that if $[\gamma]=\left[\gamma^{\prime}\right]$ then also $\left[M_{\gamma}\right]=\left[M_{\gamma^{\prime}}\right]$. If $[\gamma]=x$, we write $M_{x}$ in place of $\left[M_{\gamma}\right]$. Let $p: E \rightarrow B$ be a surface bundle with monodromy in $\mathcal{I}_{g}$ and let $\rho: \pi_{1} B \rightarrow \mathcal{I}_{g}$ be the monodromy. By post-composing with $\tau: \mathcal{I}_{g} \rightarrow \wedge^{3} H / H$, we obtain a map from $\pi_{1} B$ to an abelian group, so $\tau \circ \rho$ factors through $H_{1}(B)$. By an abuse of notation we will write $\tau(b)$ for $b \in H_{1}(B)$.

This map computes (most of) the intersection form in $H_{*}(E)$. Recall the notation from Proposition 3.1: given a curve $\alpha \subset B$, there is an induced bundle $E_{\alpha}$ over $\alpha$, which determines a homology class $E_{a}$. A given $M_{\gamma}$ can be intersected with $E_{\alpha}$ to yield a surface $\Sigma_{\alpha, \gamma}$ inside $E_{\alpha}$. Our construction will be set up so that

$$
M_{x} \cdot M_{y} \cdot M_{z} \cdot E_{b}=\tau(b)(x \wedge y \wedge z),
$$

possibly up to a sign. This is the sense in which $M_{x} \cdot M_{y} \cdot M_{z}$ is partially computable. As an aside, the intersections $M_{x} \cdot M_{y} \cdot M_{z} \cdot X$ for arbitrary $X \in H_{3} E$ will all involve intersections with further $M_{w}$ and are describable (at least in the case of bundles with section) in terms of the higher Johnson invariants

$$
\tau: H_{i}\left(\mathcal{I}_{g, *}\right) \rightarrow \bigwedge^{i+2} H,
$$

but we will not pursue this point of view further in this paper.

The construction As usual, let $\pi: E \rightarrow B$ be a surface bundle over a surface with monodromy $\rho: \pi_{1} B \rightarrow \mathcal{I}_{g}$. We turn now to the question of constructing suitable homology classes $M_{x} \in H_{3}(E)$ for $x \in H_{1}\left(\Sigma_{g}\right)$. The construction will be a higherdimensional analogue of the construction of tube-and-cap surfaces given in the previous subsection. The reader may find it helpful to consult Figure 2 as they read this subsection.

When the base space $B$ has dimension 2, a new layer of complexity is introduced by the potential absence of sections $\sigma: B \rightarrow E$, which will require some additional preparatory work in order to construct geometric representatives for homology classes. Our construction method proceeds by exploiting the fact that it is always possible to find sections defined on $B^{\prime}:=\overline{B \backslash D^{2}}$. We define $E^{\prime}:=\pi^{-1}\left(B^{\prime}\right)$ and refer to a section $\sigma: B^{\prime} \rightarrow E^{\prime}$ as a partial section of the bundle $E$. We say that two sections $\sigma_{0}$ and $\sigma_{1}$ of a fiber bundle are homotopic through sections if there exists a homotopy $\sigma_{t}$ between $\sigma_{0}$ and $\sigma_{1}$ such that $\sigma_{t}$ is a section for each fixed $t$. 

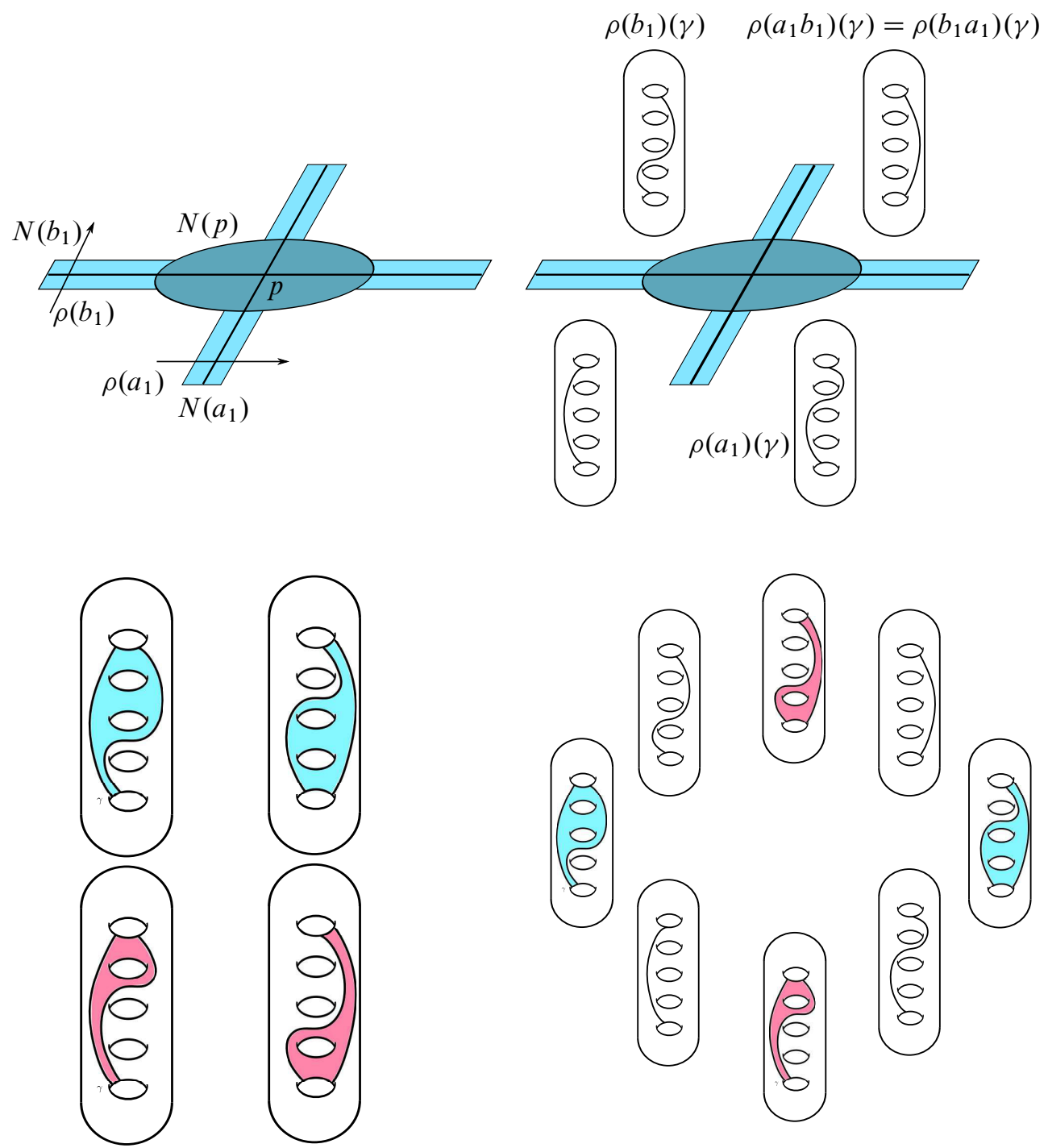

Figure 2: Upper left: the neighborhoods $N(e)$ and $N(p)$. Upper right: $M_{\gamma}^{1}$ intersected with four different fibers. Lower left: cap surfaces, lying over different portions of $N$. Lower right: a depiction of $M_{\gamma}^{2} \cap \pi^{-1}(\partial N)$.

Lemma 5.3 Let $\pi: E \rightarrow \Sigma_{h}$ be a surface bundle over a surface with monodromy $\rho: \pi_{1} \Sigma_{h} \rightarrow \operatorname{Mod}\left(\Sigma_{g}\right)$. Let $E^{\prime}=\pi^{-1}\left(\overline{\Sigma_{h} \backslash D^{2}}\right)$ and note that $\pi$ restricts to give $E^{\prime}$ the structure of a $\Sigma_{g}$-bundle over $\overline{\Sigma_{h} \backslash D^{2}}$. Then there is a one-to-one correspondence between the set of classes of partial sections $\sigma: \overline{\Sigma_{h} \backslash D^{2}} \rightarrow E^{\prime}$, up to homotopy through sections, and homomorphisms $\tilde{\rho}: F_{2 h} \rightarrow \operatorname{Mod}\left(\Sigma_{g, *}\right)$ making the diagram 
below commute:

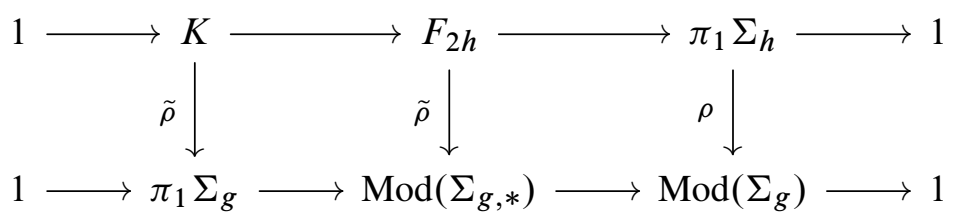

Proof This follows immediately from the well-known fact that there is a homotopy equivalence

$$
K\left(\operatorname{Mod}\left(\Sigma_{g, *}\right), 1\right) \simeq B\left(\operatorname{Diff}\left(\Sigma_{g}, *\right)\right),
$$

the latter space being the classifying space of $\Sigma_{g}$-bundles with section.

The kernel $K \triangleleft F_{2 h}$ is normally generated by a single element $\omega$, represented geometrically by the boundary of $\overline{\Sigma_{h} \backslash D^{2}}$. The element $\tilde{\rho}(\omega) \in \pi_{1} \Sigma_{g}$ associated to a section $\sigma$ will be denoted by $\omega_{\sigma}$. It is called the index curve. The following lemma is immediate from the definitions.

Lemma 5.4 Assume the notation of Lemma 5.3. Let $\sigma$ be a partial section of $E$ and let $\omega_{\sigma} \in \pi_{1} \Sigma_{g}$ be the corresponding index curve. Then there exists a local trivialization of $E$,

$$
t: \pi^{-1}\left(D^{2}\right) \rightarrow D^{2} \times \Sigma_{g},
$$

relative to which $\sigma\left(\partial D^{2}\right)$ is in the free homotopy class of $\omega_{\sigma}$.

The next lemma will be used in the course of the construction in Proposition 5.6.

Lemma 5.5 Let $S \subset \Sigma_{g} \times S^{1}$ be an embedded, closed, oriented subsurface. Suppose $\gamma: S^{1} \rightarrow \Sigma_{g} \times S^{1}$ is a section of the projection $\Sigma_{g} \times S^{1} \rightarrow S^{1}$ and that $p_{*}[\gamma]=0 \in H_{1}\left(\Sigma_{g}, \mathbb{Z}\right.$ ) (where $p: \Sigma_{g} \times S^{1} \rightarrow \Sigma_{g}$ is the obvious projection). Let $i: \Sigma_{g} \times S^{1} \rightarrow \Sigma_{g} \times D^{2}$ be the natural inclusion. If the algebraic intersection number is $[\gamma] \cdot[S]=0$ (computed in $\Sigma_{g} \times S^{1}$ ), then there exists an oriented, properly embedded 3-manifold $M \subset \Sigma_{g} \times D^{2}$ such that $\partial M=S$.

Proof The first step is to establish that $i_{*}[S]=0$ in $H_{2}\left(\Sigma_{g} \times D^{2}\right)$. The Künneth formula establishes natural splittings

$$
\begin{aligned}
& H_{1}\left(\Sigma_{g} \times S^{1}\right) \approx H_{1}\left(\Sigma_{g}\right) \oplus H_{1}\left(S^{1}\right), \\
& H_{2}\left(\Sigma_{g} \times S^{1}\right) \approx H_{2}\left(\Sigma_{g}\right) \oplus\left(H_{1}\left(\Sigma_{g}\right) \otimes H_{1}\left(S^{1}\right)\right) .
\end{aligned}
$$

In these coordinates, the map $i_{*}: H_{2}\left(\Sigma_{g} \times S^{1}\right) \rightarrow H_{2}\left(\Sigma_{g} \times D^{2}\right) \approx H^{2}\left(\Sigma_{g}\right)$ is given simply by projection onto the $H_{2}\left(\Sigma_{g}\right)$ factor. The assumptions on $\gamma$ imply that $[\gamma]$ 
generates $H_{1}\left(S^{1}\right) \leq H_{1}\left(\Sigma_{g} \times S^{1}\right)$. Under the intersection pairing, $H_{1}\left(S^{1}\right)$ is orthogonal to $H_{1}\left(\Sigma_{g}\right) \otimes H_{1}\left(S^{1}\right)$. From the assumption $[\gamma] \cdot[S]=0$, it then follows easily that $i_{*}[S]=0$. Consequently, there exists a 3-chain $C_{p}$ in $\Sigma_{g} \times D^{2}$ with $\partial C_{p}=S$.

It remains to explain why $C_{p}$ can be replaced with a smooth, oriented, properly embedded 3-manifold. This will follow from general results on representing (relative) codimension- 1 homology classes by smooth submanifolds (with boundary). The argument proceeds along very similar lines to the construction of embedded cap surfaces in fibered 3-manifolds described above. For an oriented manifold $X$ with boundary, Lefschetz duality gives an isomorphism

$$
H_{n-1}(X, \partial X, \mathbb{Z}) \approx H^{1}(X, \mathbb{Z}) \approx\left[X, S^{1}\right]
$$

In our setting, the surface $S \subset \Sigma_{g} \times S^{1}$ is represented by a map

$$
f: \Sigma_{g} \times S^{1} \rightarrow S^{1}
$$

such that $S=f^{-1}(*)$ for some regular value $* \in S^{1}$. Similarly, the (relative) homology class of $C_{p}$ in $H_{3}\left(\Sigma_{g} \times D^{2}, \Sigma_{g} \times S^{1}, \mathbb{Z}\right)$ corresponds to a map

$$
F: \Sigma_{g} \times D^{2} \rightarrow S^{1} \text {. }
$$

Moreover, as $\partial C_{p}=S$, they represent the same homology class in $H_{2}\left(\Sigma_{g} \times S^{1}, \mathbb{Z}\right)$. This means that the maps $f$ and $\left.F\right|_{\Sigma_{g} \times S^{1}}$ are homotopic. We can therefore concatenate this homotopy with $F$ to obtain a map

$$
\widetilde{F}: \Sigma_{g} \times D^{2} \rightarrow S^{1} .
$$

On the boundary, $\widetilde{F}$ equals $f$ and is therefore transverse to $* \subset S^{1}$. In order to replace $C_{p}$ by a smooth submanifold such that $\partial C_{p}=C$, we must therefore perturb $\widetilde{F}$ away from a neighborhood of $\partial\left(\Sigma_{g} \times D^{2}\right)$ and make the result everywhere transverse to $* \subset S^{1}$. The extension theorem (see [4, page 72]) asserts that we can do precisely this.

The theory of index curves established above will allow us to construct embedded representatives of homology classes in surface bundles over surfaces when suitable conditions on the monodromy are satisfied.

Proposition 5.6 Let $\pi: E \rightarrow B$ be a surface bundle over a surface with monodromy $\rho: \pi_{1} B \rightarrow \mathcal{I}_{g}$ contained in the Torelli group. Suppose there is a partial section $\sigma: B^{\prime} \rightarrow E^{\prime}$ for which the associated index curve $\omega_{\sigma}$ lies in the commutator subgroup $\left[\pi_{1} \Sigma_{g}, \pi_{1} \Sigma_{g}\right]$. Then there is an embedding

$$
\iota: H_{1}(F, \mathbb{Z}) \rightarrow H_{3}(E, \mathbb{Z})
$$


constructed so that, if $c \in H_{1}(F, \mathbb{Z})$ is a primitive class, then $\iota(c)$ can be represented by some embedded, oriented, piecewise-smooth 3-submanifold $M_{c}$ of $E$.

Proof Let $c \in H_{1}(F, \mathbb{Z})$ be given. By assumption, $c$ is primitive, so that there exists a simple closed curve $\gamma \subset \Sigma_{g}$ with $[\gamma]=c$. We will use this to construct a 3-manifold $M_{\gamma}$.

Consider a cell decomposition

$$
B=B^{0} \subset B^{1} \subset B^{2}
$$

of $B$, where $B^{0}$ consists of the single point $p$, there are $2 g$ one-cells $\left\{a_{1}, b_{1}, \ldots, a_{h}, b_{h}\right\}$ and a single two-cell $D$. For each one-cell $e$, there is an associated element $\rho(e)$ of the monodromy such that the effect of transporting a curve $\gamma$ across $e$ (from the negative to the positive side, relative to orientations of $B$ and $e$ ) sends the isotopy class of $\gamma$ to $\rho(e) \gamma$. For a one-cell $e$, let $N(e) \approx e \times I$ be a (closed) regular neighborhood in $B$. We also let $N(p)$ be a small closed neighborhood of $p$. If necessary, shrink $N(e)$ so that

$$
N:=N\left(a_{1}\right) \cup \cdots \cup N\left(b_{h}\right) \backslash N(p)
$$

is a union of $2 h$ disjoint rectangles.

Let $\gamma \subset F$ be a simple closed curve on a fiber $F$ over a point in

$$
D^{\prime}:=\overline{D \backslash\left(N(p) \cup N\left(a_{1}\right) \cup \cdots \cup N\left(b_{h}\right)\right)} .
$$

By construction, $D^{\prime}$ is nothing more than a closed disk (in the upper-left portion of Figure 2, $D^{\prime}$ is the closure of the complement of the shaded regions). The submanifold $M_{\gamma}$ will be constructed in three stages, denoted by $M_{\gamma}^{i}$ for $i=1,2,3$ : first over $D^{\prime}$, then over $N$ and finally over $N(p)$. Choose a trivialization $\pi^{-1}\left(D^{\prime}\right) \approx D^{\prime} \times F$ and define $M_{\gamma}^{1}=\gamma \times D^{\prime}$ relative to this trivialization. Then $\partial\left(M_{\gamma}^{1}\right) \subset \pi^{-1}\left(\partial D^{\prime}\right)$. We specify an orientation on $M_{\gamma}^{1}$ as follows: a point $x \in M_{\gamma}^{1}$ has a decomposition of the tangent space

$$
T_{x} M_{\gamma}^{1} \approx T_{\pi(x)} B \oplus T_{x} \gamma
$$

Both of these two summands carry pre-existing orientations and $M_{\gamma}^{1}$ is then oriented by specifying the above isomorphism to be orientation-preserving. By analogy with the construction of tube surfaces, we refer to $M_{\gamma}^{1}$ as the tube region of $M_{\gamma}$.

Next we construct $M_{\gamma}^{2}$. Let $e$ be a one-cell and consider $M_{\gamma}^{1} \cap \pi^{-1}(N(e) \cap N)$. The base space $N(e) \cap N$ is just a rectangle, so the bundle $\pi^{-1}(N(e) \cap N)$ is trivializable. We can therefore find a diffeomorphism

$$
\psi: \pi^{-1}(N(e) \cap N) \approx I \times I \times \Sigma_{g}
$$


under which $M_{\gamma}^{1} \cap \pi^{-1}(N(e) \cap N)$ is identified with

$$
(I \times\{0\} \times \gamma) \cup\left(I \times\{1\} \times \gamma^{\prime}\right),
$$

where $\gamma^{\prime}$ is some curve in the isotopy class of $\rho(e)(\gamma)$. As we saw in the previous subsection, for each $e$ there exists a family of properly embedded surfaces $S_{e}$ in $I \times \Sigma_{g}$ such that $\partial S_{e}=\{0\} \times \gamma \cup\{1\} \times \gamma^{\prime}$.

Our choice of $S_{e}$ will be dictated by the section $\sigma$. Applying $\psi$, the image of $\sigma$ in $\{t\} \times I \times \Sigma_{g}$ is a properly embedded arc $\alpha_{\sigma}$. This determines a preferred homology class in $H_{2}\left(I \times \Sigma_{g}, \partial\left(I \times \Sigma_{g}\right), \mathbb{Z}\right)$ among the set of possible $S_{e}$, by the relation $\left[\alpha_{\sigma}\right] \cdot\left[S_{e}\right]=0$.

Let $S_{e}$ be any properly embedded subsurface of $I \times \Sigma_{g}$ satisfying the conditions $\partial S_{e}=\{0\} \times \gamma \cup\{1\} \times \gamma^{\prime}$ and $\left[\alpha_{\sigma}\right] \cdot\left[S_{e}\right]=0$. We can then fill in $\pi^{-1}(N(e) \cap N)$ with $I \times S_{e}$ for each $e$, creating $M_{\gamma}^{2}$. As in the case of a tube surface, the orientation for $M_{\gamma}^{1}$ can be extended over each of these pieces coherently. We refer to $M_{\gamma}^{2} \backslash M_{\gamma}^{1}$ as the cap region of $M_{\gamma}$.

It therefore remains to construct $M_{\gamma}^{3}=M_{\gamma}$. By construction, $\partial M_{\gamma}^{2} \subset \pi^{-1}(\partial N(p))$. We would like to be able to fill this boundary in by inserting a "plug" contained in $\pi^{-1}(N(p))$. A priori, there is a homological obstruction to this: if $\left[\partial M_{\gamma}^{2}\right] \neq 0$ in $H_{2}\left(\pi^{-1}(N(p))\right)$ then this problem is not solvable even on the chain level.

However, the assumption that the index curve $\omega_{\sigma}$ is in $\left[\pi_{1} \Sigma_{g}, \pi_{1} \Sigma_{g}\right]$ will imply that this obstruction vanishes. Let $t: \pi^{-1}(N(p)) \rightarrow D^{2} \times \Sigma_{g}$ be the trivialization of Lemma 5.4 and define $\gamma=t(\sigma(\partial(N(p))))$. Set $S=t\left(\partial\left(M_{\gamma}^{2}\right)\right)$. By Lemma 5.4, $[\gamma]=0 \in H_{1}\left(\pi^{-1}(N(p))\right) \approx H_{1}\left(\Sigma_{g}\right)$. We wish to show that $[\gamma] \cdot[S]=0$. By construction, $\partial\left(M_{\gamma}^{2}\right)$ consists of $4 g$ subsurfaces, corresponding to the $2 g$ surfaces $S_{a_{1}}, \ldots, S_{b_{g}}$, each appearing twice (once for each component of $N(e) \cap N(p)$ ). Similarly, $\gamma$ is comprised of $4 g$ segments, again indexed by the components of $N(e) \cap N(p)$. On each one of these components, the relevant $S_{e}$ was selected to have zero algebraic intersection with the relevant portion of $\gamma$, so the same holds true globally: $[\gamma] \cdot[S]=0$.

Applying Lemma 5.5, we obtain a 3-manifold $M_{p} \subset N(p) \times \Sigma_{g}$ with $\partial M_{p}=t\left(\partial\left(M_{\gamma}^{2}\right)\right)$. Extending the orientation of $M_{\gamma}^{2}$ over $M_{p}$, the result is an oriented, piecewise-smooth submanifold $M_{\gamma} \subset E$.

Remark 5.7 It is apparent in the above construction that if $\gamma$ and $\gamma^{\prime}$ are homologous curves, the associated 3-manifolds $M_{\gamma}$ and $M_{\gamma^{\prime}}$ are homologous. Accordingly, if $[\gamma]=\left[\gamma^{\prime}\right]=x$, we adopt the notation $M_{x}=\left[M_{\gamma}\right]=\left[M_{\gamma^{\prime}}\right]$.

While, in general, not every surface bundle over a surface satisfies the hypotheses of Proposition 5.6 (specifically the requirement that there exist a partial section with 
$\left.\left[\omega_{\sigma}\right]=0 \in H_{1}\left(\Sigma_{g}, \mathbb{Z}\right)\right)$, it turns out that this is always the case for surface bundles over surfaces with monodromy in $\mathcal{K}_{g}$.

Lemma 5.8 Let $\rho: \pi_{1} \Sigma_{h} \rightarrow \mathcal{K}_{g}$ be given. Then, for any lift $\tilde{\rho}: F_{2 h} \rightarrow \mathcal{K}_{g, *}$ of $\rho$, the index curve satisfies $\omega_{\sigma} \in\left[\pi_{1} \Sigma_{g}, \pi_{1} \Sigma_{g}\right]$.

Proof When restricted to $\mathcal{K}_{g}$, the Birman exact sequence takes the form

$$
1 \rightarrow\left[\pi_{1} \Sigma_{g}, \pi_{1} \Sigma_{g}\right] \rightarrow \mathcal{K}_{g, *} \rightarrow \mathcal{K}_{g} \rightarrow 1
$$

The result follows.

An essential feature of the above construction is the relationship between an $M_{\gamma}$ and a sub-bundle $E_{\alpha}$ lying over a curve $\alpha \subset B$. Suppose $\alpha$ is chosen so that, relative to the cell decomposition of $B$ used in constructing $M_{\gamma}, \alpha$ is transverse to all the one-cells $e$ and does not pass through $N(p)$. Then a little visual imagination reveals that the intersection of $M_{\gamma}$ and $E_{\alpha}$ is given by a tube surface for $\gamma$ sitting inside $E_{\alpha}$. We call the resulting surface $\Sigma_{\alpha, \gamma}$ and then $\left[\Sigma_{\alpha, \gamma}\right]$ is denoted by $\Sigma_{a, x}$, where $[\alpha]=a$ and $[\gamma]=x$.

We define a family of $M_{x}$ to be a choice of $M_{x}$ for each $x \in H_{1}(F)$ such that, for all $c \in \mathbb{Z}$ and $x, y \in H_{1}(F)$,

$$
M_{c x+y}=c M_{x}+M_{y} .
$$

Different choices of $M_{x}$ lead to different spaces of $\Sigma_{b, x}$ but, conversely, a choice of a family of $M_{x}$ leads to a corresponding distinguished summand of $H_{2}(E)$.

\subsection{Determination of the intersection form}

From this point onwards, we assume without further comment that our surface bundle over a surface, $\pi: E \rightarrow B$, satisfies the hypotheses of Proposition 5.6 (as a special case, these results apply to all surface bundles over surfaces with monodromy in $\mathcal{K}_{g}$, by Lemma 5.8). The purpose of this subsection is to give a description of the cup product structure on $H^{*}(E, \mathbb{Z})$; equivalently, we will describe the intersection form. By Poincaré duality, it suffices to determine, for each $X$, the set of pairings $X \cdot Y$.

Proposition 5.9 Let $i_{B}$ and $i_{F}$ denote the algebraic intersection pairing on the homology of the base and on the fiber, respectively.

(1) There exists a unique class $C \in H_{2}(E)$ such that $C \cdot[F]=1$ and $C \cdot \Sigma_{b, z}=0$ for all $b \in H_{1}(B)$ and $z \in H_{1}\left(\Sigma_{g}\right)$. The intersection pairing $H_{2}(E) \otimes H_{2}(E) \rightarrow \mathbb{Z}$ 
is given as follows, where $e=C^{2}$ by definition:

\begin{tabular}{c|ccc} 
& $C$ & {$[F]$} & $\Sigma_{a, z}$ \\
\hline$C$ & $e$ & 1 & 0 \\
{$[F]$} & 1 & 0 & 0 \\
$\Sigma_{b, w}$ & 0 & 0 & $-i_{B}(a, b) i_{F}(z, w)$
\end{tabular}

In the case where the monodromy is contained in the Johnson kernel, we have $e=0$.

(2) For any family of $M_{x}$, we have

$$
\begin{aligned}
E_{a} \cdot E_{b} & =i_{B}(a, b)[F], \\
M_{x} \cdot E_{b} & =\Sigma_{b, x}, \\
M_{z} \cdot M_{w} \cdot[F] & =i_{F}(z, w) .
\end{aligned}
$$

(3) Let $\sigma: B^{\prime} \rightarrow E^{\prime}$ be a partial section for which $\left[\omega_{\sigma}\right]=0 \in H_{1}(F)$. Associated to such a section is a lift of $\tau: H_{1}(B) \rightarrow \wedge^{3} H / H$ to $\tilde{\tau}: H_{1}(B) \rightarrow \bigwedge^{3} H$. The choice of $\sigma$ gives rise to a splitting

$$
H_{3}(E)=\pi^{!}\left(H_{1}(B)\right) \oplus H_{1}(M)=\left\{E_{b}, b \in H_{1}(B)\right\} \oplus\left\{M_{z}, z \in H_{1}(F)\right\}
$$

relative to which

$$
M_{x} \cdot M_{y} \cdot M_{z} \cdot E_{b}=M_{x} \cdot M_{y} \cdot \Sigma_{b, z}=\tilde{\tau}(b)(x \wedge y \wedge z) .
$$

In the case where the monodromy is contained in the Johnson kernel, we can take the canonical lift $\tilde{\tau} \equiv 0$ and, for this family of $M_{x}$, we have

$$
C \cdot M_{x}=0 \text { and } C^{2}=0
$$

for all $x \in H_{1}\left(\Sigma_{g}\right)$.

Remark The intersection pairing $H_{n-k} E \otimes H_{k} E \rightarrow \mathbb{Z}$ identifies $H_{n-k} E$ with $\operatorname{Hom}\left(H_{k} E, \mathbb{Z}\right)$ and hence with $H^{k} E$ by the universal coefficients theorem, since the homology of a surface bundle over a surface with monodromy in $\mathcal{I}_{g}$ is torsion-free (see Proposition 3.1). Therefore, Proposition 5.9 can also be viewed as a description of the cup product in $H^{*}(E)$.

Proof Before beginning the proof of the statements, a comment on orientations is in order. Recall that if $X$ and $Y$ are embedded surfaces intersecting transversely, then $X \cap Y$ is oriented via the convention that

$$
N(X) \oplus N(Y) \oplus T(X \cap Y)
$$


should be positively oriented, where, for $W=X$ or $W=Y, N(W)$ is oriented by the convention that $N(W) \oplus T(W)$ be positively oriented with respect to the orientation fixed on $W$. Note that relative to this convention, if $X$ is of odd codimension, then $X \cdot X=0$; we will often employ this fact without comment in the sequel.

Recall that the submanifolds $\Sigma_{x} \subset M_{\phi}$ and $M_{z} \subset E$ have been oriented using a "base first" convention; see (3) and (4). As remarked already in the proof of Proposition 4.1, $E$ itself is oriented by selecting orientations for $B$ and $F$. It is a somewhat tedious process to go through and verify the signs on all of the intersections being asserted in this theorem, so we omit the full verification of these results. At the same time, the reader who is interested in verifying the calculations should have no trouble doing so by carefully tracking the orientation conventions we have laid out.

It will turn out to be most natural to construct $C$ after verifying the statements not involving $C$. We begin with computing $\Sigma_{a, z} \cdot \Sigma_{b, w}$. These are represented by surfaces contained in some $E_{\alpha}$ and $E_{\beta}$, respectively, where they are tube surfaces constructed from curves $\gamma$ and $\delta$. We can arrange it so that $\alpha$ and $\beta$ intersect transversely and such that, over these points, the surfaces intersect in their tube regions. Following the orientation conventions as above, one verifies that the local intersection at such a point $(p, q)$, written $I_{(p, q)}$, is equal to $-I_{p} I_{q}$, where $I_{p}$ denotes the local intersection of $\alpha$ and $\beta$ relative to the orientation on $B$ and $I_{q}$ is the local intersection of $\gamma$ and $\delta$ relative to the orientation on $F$. Summing over all local intersections gives the result in the lower right-hand corner of the table in Proposition 5.9(1).

The relation $[F] \cdot \Sigma_{a, z}=0$ is easy to verify, by taking $[F]$ to be represented by a fiber not contained in the $E_{\alpha}$ containing $\Sigma_{a, z}$. This same idea also shows $[F]^{2}=0$, by picking representative fibers over distinct points.

Let us turn now to Proposition 5.9(2). If $E_{\alpha}$ and $E_{\beta}$ intersect transversely at a point, then $E_{\alpha} \cap E_{\beta}=F$, the fiber over the point of intersection; a check of the orientation conventions shows that the orientation on $F$ given by the intersection convention agrees with the predetermined orientation, so that

$$
E_{a} \cdot E_{b}=i_{B}(a, b)[F]
$$

as asserted.

The manifolds $M_{\gamma}$ were constructed so as to intersect each $E_{b}$ in a tube surface, so the relation

$$
M_{z} \cdot E_{b}=\Sigma_{b, z}
$$

can be taken as a definition of the orientation on $\Sigma_{b, z}$. We choose this over the alternative because it can be verified that, under this convention, the orientation on $\Sigma_{b, z}$ agrees with the "base first" convention discussed above. 
Now let $M_{x}$ and $M_{y}$ be given and consider $M_{x} \cdot M_{y} \cdot[F]$. By perturbing the oneskeleton of $B$, it can be arranged so that the plugs for $M_{x}$ and $M_{y}$ are disjoint, the cap regions intersect transversely and the representative fiber intersects $M_{x}$ and $M_{y}$ in their tube regions. The local picture therefore becomes the intersection of $x$ and $y$ on $F$. A check of the orientation convention then shows

$$
M_{x} \cdot M_{y} \cdot[F]=i_{F}(x, y) .
$$

Turning to Proposition 5.9(3), consider now a four-fold intersection

$$
M_{x} \cdot M_{y} \cdot M_{z} \cdot\left[E_{\beta}\right] .
$$

We will assume without further comment that the intersection of representative submanifolds has been made suitably transverse by choosing one-skeleta wisely. The $M_{w}$ were constructed so that the problem of computing $M_{x} \cdot M_{y} \cdot M_{z} \cdot\left[E_{\beta}\right]$ is exactly the same as the problem of computing the corresponding $\Sigma_{x} \cdot \Sigma_{y} \cdot \Sigma_{z}$ inside the 3-manifold $E_{\beta}$, up to a sign which records whether the orientation on $M_{x} \cdot\left[E_{\beta}\right]$ agrees with the orientation on the corresponding $\Sigma_{x} \subset E_{\beta}$; the convention $M_{x} \cdot E_{b}=\Sigma_{x, b}$ makes this sign positive. Lemma 5.2 shows that, within $E_{b}$, there exist choices of homology classes $\Sigma_{x}$ such that

$$
\Sigma_{x} \cdot \Sigma_{y} \cdot \Sigma_{z}=\tilde{\tau}(b)(x \wedge y \wedge z) .
$$

Recall from Lemma 5.2 that the $\Sigma_{x}$ are obtained by starting with an arbitrary family $\Sigma_{x}^{\prime}$ and adding appropriate multiples of $[F]$. By the preceding, if $a \in B$ satisfies $i_{B}(a, b)=1$, then

$$
\left(M_{z}+E_{a}\right) \cdot E_{b}=M_{z} \cdot E_{b}+[F] .
$$

This shows that, by adding appropriate multiples of $E_{a}$ to $M_{z}$ (as specified by the formulas in Lemma 5.2), for a given $b$ the formula

$$
M_{x} \cdot M_{y} \cdot M_{z} \cdot\left[E_{\beta}\right]=\tilde{\tau}(b)(x \wedge y \wedge z)
$$

can be made to hold. By choosing a symplectic basis for $H_{1}(B)$, this can be made to hold for all $b \in H_{1}(B)$ simultaneously.

It therefore remains to construct the class $C$. If $x, y \in H_{1}\left(\Sigma_{g}\right)$ satisfy $i_{F}(x, y)=1$, then $[F] \cdot M_{x} \cdot M_{y}=1$. Similarly, if $\alpha$ and $\beta$ are loops in $B$ intersecting transversely exactly once and $M_{x}$ and $M_{y}$ are as above, then

$$
\Sigma_{\alpha, x} \cdot \Sigma_{\beta, y}=\Sigma_{\alpha, x} \cdot M_{x} \cdot E_{\beta}= \pm 1
$$

As the space spanned by $[F]$ and the $\Sigma_{b, x}$ classes has codimension one in $H_{2}(E)$, (5) and (6) together show that the space of classes in $H_{2}(E)$ pairing trivially with the 
space of $M_{x}$ has dimension at most one. We claim that

$$
C=M_{x_{1}} \cdot M_{y_{1}}+\sum_{(b, z) \in \mathcal{B} \times \mathcal{F}} \tilde{\tau}(b)\left(x_{1} \wedge y_{1} \wedge z\right) \Sigma_{\hat{b} \hat{z}}
$$

has all the required properties; here, $\mathcal{B}$ and $\mathcal{F}$ are symplectic bases for $H_{1}(B)$ and $H_{1}(F)$, respectively, the map $x \mapsto \hat{x}$ satisfies $i(x, \hat{x})=1, x_{1} \in \mathcal{B}$ and $\hat{x}_{1}=y_{1}$. Recall that $C$ is asserted to have the following properties: $C \cdot[F]=1$ and $C \cdot \Sigma_{b, z}=0$ for all $b \in H_{1}(B)$ and $z \in H_{1}\left(\Sigma_{g}\right)$. Additionally, when the monodromy of $E$ is contained in the Johnson kernel, we require $C^{2}=0$ and $C \cdot M_{x}=0$ for $M_{x}$ in the family associated to the lift of $\tau$ to the zero homomorphism. The proof is a direct calculation. For $C \cdot[F]$, one has, by Proposition 5.9(1) and then Proposition 5.9(2),

$$
C \cdot[F]=\left(M_{x_{1}} \cdot M_{y_{1}}+\sum_{(b, z) \in \mathcal{B} \times \mathcal{F}} \tilde{\tau}(b)\left(x_{1} \wedge y_{1} z\right) \Sigma_{\hat{b} \hat{z}}\right) \cdot[F]=M_{x_{1}} \cdot M_{y_{1}} \cdot[F]=1 .
$$

Computation of $C \cdot \Sigma_{b, z}$ proceeds by Proposition 5.9(3) and Proposition 5.9(1), respectively:

$$
\begin{aligned}
C \cdot \Sigma_{b, z} & =M_{x_{1}} \cdot M_{y_{1}} \cdot \Sigma_{b, z}+\tilde{\tau}(b)\left(x_{1} \wedge y_{1} \wedge z\right)\left(\Sigma_{\hat{b} \hat{z}}\right) \cdot \Sigma_{b, z} \\
& =\tilde{\tau}(b)\left(x_{1} \wedge y_{1} \wedge z\right)-\tilde{\tau}(b)\left(x_{1} \wedge y_{1} \wedge z\right) \\
& =0 .
\end{aligned}
$$

When the monodromy of $E$ is contained in $\mathcal{K}_{g}$, the above formula for $C$ simplifies to $C=M_{x_{1}} \cdot M_{y_{1}}$, from which it is apparent that $C^{2}=0$. To see that $C \cdot M_{x}=0$ for all $x$, we will apply Poincaré duality to see that it suffices to show that

$$
C \cdot M_{x} \cdot Y=0
$$

for all classes $Y \in H_{3} E$. Since $M_{x} \cdot E_{b}=\Sigma_{b x}$ and we have shown $C \cdot \Sigma_{b x}=0$, it remains only to consider $C \cdot M_{z} \cdot M_{w}$. Expanding $M_{z} \cdot M_{w}$ in the additive basis for $\mathrm{H}_{2}(E)$,

$$
M_{z} \cdot M_{w}=\alpha[F]+\beta C+\sum_{(b, z) \in \mathcal{B} \times \mathcal{F}} \gamma_{b, z} \Sigma_{\hat{b}, \hat{z}}
$$

As the monodromy of $E$ is contained in $\mathcal{K}_{g}$, we have $M_{z} \cdot M_{w} \cdot \Sigma_{b, x}=0$; applying this in coordinates for some $(b, x) \in \mathcal{B} \times \mathcal{F}$ gives, by applying the prior formulas,

$$
0=\left(\alpha[F]+\beta C+\sum_{(b, z) \in \mathcal{B} \times \mathcal{F}} \gamma_{b, z} \Sigma_{\hat{b}, \hat{z}}\right) \cdot \Sigma_{b, x}=-\gamma_{b, x}
$$

so that all $\gamma_{b, z}$ are 0 . Consequently, $M_{z} \cdot M_{w}=\alpha[F]+\beta C$. Recalling that $[F]^{2}=$ $C^{2}=0$ and that $\left(M_{z} \cdot M_{w}\right)^{2}=0$, this implies $\alpha \beta=0$. 
Also,

$$
i_{F}(z, w)=M_{z} \cdot M_{w} \cdot[F]=\beta .
$$

Therefore, we conclude that, in the case $i_{F}(z, w) \neq 0$,

$$
M_{z} \cdot M_{w}=i_{F}(z, w) C .
$$

As $C^{2}=0$, this shows the result in this case. Now suppose that $i_{F}(z, w)=0$. Then we can find $z^{\prime}$ such that $M_{z} \cdot M_{z^{\prime}}=c C$ by the above, with $c \neq 0$, then

$$
0=M_{z} \cdot M_{w} \cdot M_{z} \cdot M_{z^{\prime}}=c M_{z} \cdot M_{w} \cdot C .
$$

This shows that $M_{z} \cdot M_{w} \cdot C=0$ for all $z$ and $w$, finishing the proof of Proposition 5.9.

\section{Multisections and splittings on rational cohomology}

Let $p: E \rightarrow B$ be a surface bundle over an arbitrary base space $B$ equipped with a section $\sigma: B \rightarrow E$. Then there is an associated splitting of $H^{1}(E, \mathbb{Z})$ as a direct sum,

$$
H^{1}(E, \mathbb{Z})=\operatorname{Im} p^{*} \oplus \operatorname{ker} \sigma^{*} .
$$

The condition that $p: E \rightarrow B$ admit a section is restrictive. However, recent work of Hamenstädt shows that all surface bundles over surfaces with zero signature admit multisections (see Theorem 6.2). In this section, we develop some necessary machinery showing how a multisection of a surface bundle gives rise to a splitting of $H^{1}(E, \mathbb{Q})$, similarly to (7). The results of this section will be required in the proof of Theorem 1.1.

Remark 6.1 Theorem 6.2 is the only result in this section that requires the base space $B$ to be a surface of genus $g \geq 2$. Lemma 6.3 and Proposition 6.4 are valid for any base space $B$.

Let $\operatorname{Conf}_{n}(E)$ denote the configuration space of $n$ unordered distinct points in $E$ and let PConf $_{n}(E)$ denote the space of $n$ ordered distinct points in $E$. The symmetric group $S_{n}$ on $n$ letters acts freely on $\operatorname{PConf}_{n}(E)$ by permuting the order of the points, and $\operatorname{PConf}_{n}(E) / S_{n}=\operatorname{Conf}_{n}(E)$.

By a multisection of $p: E \rightarrow B$, we mean a map

$$
\sigma: B \rightarrow \operatorname{Conf}_{n}(E)
$$

for some $n \geq 1$ such that the composition

$$
B \rightarrow \operatorname{Conf}_{n}(E) \rightarrow B^{n} / S_{n}
$$


is given by $x \mapsto[x, \ldots, x]$. In other words, a multisection selects $n$ distinct unordered points in each fiber. A pure multisection is a map

$$
\sigma: B \rightarrow \operatorname{PConf}_{n}(E)
$$

such that the composition

$$
B \rightarrow \operatorname{PConf}_{n}(E) \rightarrow B^{n}
$$

is given by $x \mapsto(x, \ldots, x)$. Our interest in multisections is due to the following result of Hamenstädt (see [5]; also personal communication, 2015):

Theorem 6.2 (Hamenstädt) Let $p: E \rightarrow B$ be a surface bundle over a surface such that the signature of $E$ is zero (eg a bundle with at least one fibering with monodromy lying in $\mathcal{I}_{g}$ ). Then $p: E \rightarrow B$ has a multisection $\sigma$ of cardinality $2 g-2$.

We will use this result to obtain a splitting on $H^{*}(E, \mathbb{Q})$. As $(7)$ indicates, this is straightforward when the multisection is pure; the work will be to obtain the required maps for general multisections. First note that, by taking a finite cover $\widetilde{B} \rightarrow B$, we can pull the bundle back to $\tilde{p}: \widetilde{E} \rightarrow \widetilde{B}$ so that the multisection pulls back to a pure multisection

$$
\psi: \widetilde{B} \rightarrow \operatorname{PConf}_{n}(\widetilde{E}) .
$$

Moreover, we can assume that the covering $\widetilde{B} \rightarrow B$ is normal with deck group $\Gamma$. By pulling back the $\Gamma$ action on $\widetilde{B}$, we see that $\Gamma$ also acts on $\widetilde{E}$, by sending the fiber over $b$ to the fiber over $\gamma(b)$. Then the multisection $\psi$ is in fact $\Gamma$-equivariant. This suggests the following lemma:

Lemma 6.3 Let $\widetilde{\sigma}: \widetilde{B} \rightarrow \widetilde{E}$ be a $\Gamma$-equivariant section. Then there is an induced map on $\Gamma$-invariant cohomology:

$$
\tilde{\sigma}^{*}: H^{*}(\widetilde{E}, \mathbb{Q})^{\Gamma} \rightarrow H^{*}(\widetilde{B}, \mathbb{Q})^{\Gamma} .
$$

As a result, the transfer map

$$
\tau^{*}: H^{*}(\widetilde{B}, \mathbb{Q}) \rightarrow H^{*}(B, \mathbb{Q})
$$

is injective when restricted to $\widetilde{\sigma}^{*}\left(H^{*}(\widetilde{E}, \mathbb{Q})^{\Gamma}\right)$.

Proof If $f: X \rightarrow Y$ is any $\Gamma$-equivariant map of topological spaces, then the map $f^{*}: H^{*}(Y) \rightarrow H^{*}(X)$ will be equivariant, so will restrict to a map on the $\Gamma$-invariant subspaces. Transfer (see [6]) gives an identification $H^{*}(\widetilde{B}, \mathbb{Q})^{\Gamma} \approx H^{*}(B, \mathbb{Q})$ and the remaining statement follows. 
We now come to the main result of the section. This asserts that, when $p: E \rightarrow B$ is a surface bundle with a multisection $\sigma: B \rightarrow \operatorname{Conf}_{n}(E)$, there exists a map $\hat{\sigma}^{*}: H^{*}(B, \mathbb{Q}) \rightarrow H^{*}(E, \mathbb{Q})$ with many of the same properties as (the pullback of) an actual section map.

Proposition 6.4 Suppose $\sigma: B \rightarrow \operatorname{Conf}_{n}(E)$ is a multisection. Then there exist maps

$$
\begin{gathered}
\hat{\sigma}^{*}: H^{*}(E, \mathbb{Q}) \rightarrow H^{*}(B, \mathbb{Q}), \\
\hat{\sigma}_{*}: H_{*}(B, \mathbb{Q}) \rightarrow H_{*}(E, \mathbb{Q}),
\end{gathered}
$$

with the following properties:

$$
\begin{gathered}
\hat{\sigma}^{*} \circ p^{*}: H^{*}(B) \rightarrow H^{*}(B)=\mathrm{id}, \\
p_{*} \circ \hat{\sigma}_{*}: H_{*}(B) \rightarrow H_{*}(B)=\mathrm{id} .
\end{gathered}
$$

(2) The maps $\hat{\sigma}^{*}$ and $\hat{\sigma}_{*}$ are adjoint under the evaluation pairing. That is, for all $\alpha \in H^{*}(E)$ and $x \in H_{*}(B)$,

$$
\left\langle\alpha, \hat{\sigma}_{*} x\right\rangle=\left\langle\hat{\sigma}^{*} \alpha, x\right\rangle .
$$

(3) If $\alpha \in \operatorname{ker} \hat{\sigma}^{*}$ then, for any $\beta \in H^{*}(E, \mathbb{Q})$ and any $x \in H_{*}(B, \mathbb{Q})$,

$$
\left\langle\alpha \smile \beta, \hat{\sigma}_{*}(x)\right\rangle=0 .
$$

Consequently, $\hat{\sigma}^{*}$ induces a splitting

$$
H^{1}(E, \mathbb{Q})=\operatorname{Im} p^{*} \oplus \operatorname{ker} \hat{\sigma}^{*} .
$$

Proof Begin by assuming that the multisection is pure. Let $p_{i}$ : $\operatorname{PConf}_{n}(E) \rightarrow E$ be the projection onto the $i^{\text {th }}$ coordinate for $i=1, \ldots, n$. We define

$$
\begin{aligned}
& \hat{\sigma}^{*}(\alpha)=\frac{1}{n} \sum_{i=1}^{n} \sigma^{*}\left(p_{i}^{*}(\alpha)\right), \\
& \hat{\sigma}_{*}(x)=\frac{1}{n} \sum_{i=1}^{n}\left(p_{i}\right)_{*}\left(\sigma_{*}(x)\right) .
\end{aligned}
$$

Then properties (1)-(3) follow by direct verification.

In the general case, let $c: \widetilde{B} \rightarrow B$ be a normal covering such that $\sigma$ pulls back to a pure multisection $\psi$. We will use $\bar{c}$ to denote the covering $\widetilde{E} \rightarrow E$. Let $\tau^{*}: H^{*}(\widetilde{B}, \mathbb{Q}) \rightarrow H^{*}(B, \mathbb{Q})$ be the transfer map, normalized so that $c^{*} \circ \tau^{*}=$ id. Then define $\hat{\sigma}^{*}: H^{*}(E, \mathbb{Q}) \rightarrow H^{*}(B, \mathbb{Q})$ by

$$
\widehat{\sigma}^{*}=\tau^{*} \circ \hat{\psi}^{*} \circ \bar{c}^{*} \text {. }
$$


Similarly, define $\hat{\sigma}_{*}: H_{*}(B, \mathbb{Q}) \rightarrow H_{*}(E, \mathbb{Q})$ by

$$
\widehat{\sigma}_{*}=\bar{c}_{*} \circ \widehat{\psi}_{*} \circ \tau_{*} \text {. }
$$

For what follows, it will be useful to refer to the following diagram:

By definition,

$$
\begin{aligned}
& H^{*}(\widetilde{E}) \underset{\bar{c}^{*}}{\stackrel{\tau^{*}}{\rightleftarrows}} H^{*}(E)
\end{aligned}
$$

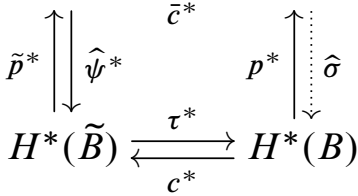

$$
\hat{\sigma}^{*} \circ p^{*}=\tau^{*} \circ \hat{\psi}^{*} \circ \bar{c}^{*} \circ p^{*} .
$$

By commutativity, $\bar{c}^{*} \circ p^{*}=\tilde{p}^{*} \circ c^{*}$. Then

$$
\tau^{*} \circ \widehat{\psi}^{*} \circ \bar{c}^{*} \circ p^{*}=\tau^{*} \circ \widehat{\psi}^{*} \circ \tilde{p}^{*} \circ c^{*}=\tau^{*} \circ c^{*}=\mathrm{id} .
$$

Here we have used the property $\hat{\psi}^{*} \circ \tilde{p}^{*}=$ id for the pure multisection $\psi$ as well as our normalization convention $\tau^{*} \circ c^{*}=\mathrm{id}$ for the transfer map. A similar calculation proves the corresponding result for $\hat{\psi}_{*}$ and (1) follows.

Statement (2) follows from the observation that the cohomology and homology transfer maps are adjoint under the evaluation pairing. That is, if $\tilde{X} \rightarrow X$ is a normal covering space with deck group $\Gamma$ then, for $x \in H_{*}(X)$ and $\alpha \in H^{*}(\tilde{X})$,

$$
\left\langle\alpha, \tau_{*}(x)\right\rangle=\left\langle\tau^{*}(\alpha), x\right\rangle .
$$

As $\hat{\psi}^{*}$ and $\bar{c}^{*}$ certainly also enjoy this adjointness property, so does $\hat{\sigma}^{*}$, and (2) follows.

To establish (3), suppose $\alpha \in \operatorname{ker} \hat{\sigma}^{*}$ and take $\beta \in H^{*}(E, \mathbb{Q})$ and $x \in H_{*}(B, \mathbb{Q})$. As the transfer map is not a ring homomorphism, (3) does not follow immediately from (2). However, we see that

$$
\left\langle\alpha \smile \beta, \hat{\sigma}_{*}(x)\right\rangle=\left\langle\hat{\sigma}^{*}(\alpha \smile \beta), x\right\rangle=\left\langle\tau^{*}\left(\left(\hat{\psi}^{*} \circ \bar{c}^{*}\right)(\alpha) \smile\left(\hat{\psi}^{*} \circ \bar{c}^{*}\right)(\beta)\right), x\right\rangle .
$$

It therefore suffices to show that $\hat{\psi}^{*} \circ \bar{c}^{*}(\alpha)=0$. This follows from Lemma 6.3. Indeed, $\bar{c}^{*}(\alpha) \in H^{*}(\widetilde{E}, \mathbb{Q})^{\Gamma}$ and $\hat{\psi}^{*}$, being a sum of $\Gamma$-equivariant maps, is itself $\Gamma$-equivariant, so $\widehat{\psi}^{*} \circ \bar{c}^{*}$ takes image in $H^{*}(\widetilde{B}, \mathbb{Q})^{\Gamma}$. On the one hand, we have

$$
0=\widehat{\sigma}^{*} \alpha=\tau^{*} \circ \widehat{\psi}^{*} \circ \bar{c}^{*}(\alpha)
$$

by assumption. Also, by Lemma $6.3, \tau^{*}$ is injective on the image of $\hat{\psi}^{*} \circ \bar{c}^{*}$, so that $\widehat{\psi}^{*} \circ \bar{c}^{*}(\alpha)=0$ as desired. 


\section{Unique fibering in the Johnson kernel}

This section is devoted to the proof of Theorem 1.1. The outline is as follows. Let $p_{1}: E \rightarrow B_{1}$ be a surface bundle with monodromy in the Torelli group $\mathcal{I}_{g}$ and suppose there is a second distinct fibering $p_{2}: E \rightarrow B_{2}$ with fiber $F_{2}$. The proof proceeds by analyzing $\left[F_{2}\right]$ in the coordinates on $H_{*}(E)$ coming from the Torelli fibering $p_{1}$. On the one hand, the intersection form in these coordinates is completely understood by virtue of Proposition 5.9. On the other, $\left[F_{2}\right]$ is realizable as an intersection of classes induced from $H_{1}\left(B_{2}\right)$. Under the assumption that the monodromy of $p_{1}$ is contained in $\mathcal{K}_{g}$ and not merely $\mathcal{I}_{g}$, it will follow that there is a unique possibility for $\left[F_{2}\right]$. The final step will be to extract the condition that the genera of $F_{2}$ and $B_{1}$ must be equal from the cohomology ring $H^{*}(E)$ and to argue that this enforces the triviality of either bundle structure.

The fundamental class of a second fiber In this subsection we will compute $\left[F_{2}\right]$ in the coordinates on $H_{2}$ coming from the fibering $p_{1}$. The results are formulated under the more general assumption that the monodromy of $p_{1}$ lie in $\mathcal{I}_{g}$ rather than $\mathcal{K}_{g}$, because we feel that the arguments are clearer in this larger context. The main objective is Lemma 7.3.

Suppose that $p_{1}: E \rightarrow B_{1}$ is a bundle with monodromy lying in $\mathcal{I}_{g}$. Suppose there is a partial section $\sigma: B^{\prime} \rightarrow E^{\prime}$ such that $\left[\omega_{\sigma}\right]=0 \in H_{1}(F)$, giving rise to a lift $\tilde{\tau}$ of the Johnson homomorphism to $\bigwedge^{3} H$; then, by Proposition 5.9(3), there is a natural splitting

$$
H_{3}(E) \approx p_{1}^{!} H_{1}\left(B_{1}\right) \oplus H_{1}\left(F_{1}\right) .
$$

We use this direct sum decomposition to define the projections

$$
P: H_{3}(E) \rightarrow p_{1}^{!} H_{1}\left(B_{1}\right) \quad \text { and } \quad Q: H_{3}(E) \rightarrow H_{1}(F)
$$

and we consider the restrictions of $P$ and $Q$ to $p_{2}^{!}\left(H_{1}\left(B_{2}\right)\right)$ for a second fibering $p_{2}: E \rightarrow B_{2}$. Where convenient, we will also define $P$ and $Q$ on $H_{1}\left(B_{2}\right)$ directly, by precomposing with the injection $p^{!}$.

Lemma 7.1 For any second fibering $p_{2}: E \rightarrow B_{2}$, the restriction of $Q$ to $H_{1}\left(B_{2}\right)$ is a symplectic mapping with respect to $d i_{F_{1}}$ on $H_{1}\left(F_{1}\right)$ and $i_{B_{2}}$ on $H_{1}\left(B_{2}\right)$, where $d=\left[F_{1}\right] \cdot\left[F_{2}\right]$ is the algebraic intersection number of the two fibers.

Proof There exist classes $x, y \in H_{1}\left(B_{2}\right)$ such that $x \cdot y=1 \in H_{0}\left(B_{2}\right)$, so that $\left[F_{2}\right]=p_{2}^{!} x \cdot p_{2}^{!} y$ and there are expressions

$$
p_{2}^{!} x=P x+Q x, \quad p_{2}^{!} y=P y+Q y .
$$


Consequently,

$$
\left[F_{2}\right]=P x \cdot P y+P x \cdot Q y-P y \cdot Q x+Q x \cdot Q y .
$$

By Proposition 5.9, $\left[F_{1}\right] \cdot P z=0$ for all $z \in H_{1}\left(B_{2}\right)$, so that

$$
d=\left[F_{1}\right] \cdot\left[F_{2}\right]=\left[F_{1}\right] \cdot Q x \cdot Q y,
$$

with the first equality holding by assumption. The condition $\left[F_{2}\right]=p_{2}^{!} x \cdot p_{2}^{!} y$ is equivalent to $i_{B_{2}}(x, y)=1$. By Proposition 5.9,

$$
d=\left[F_{1}\right] \cdot Q x \cdot Q y=i_{F_{1}}(Q x, Q y),
$$

proving the claim.

As in the above proof, let $x, y \in H_{1}\left(B_{2}\right)$ satisfy $x \cdot y=1$. By Poincaré duality, in order to determine $\left[F_{2}\right]$ it suffices to determine the collection of cup products $\left[F_{2}\right] \cdot Z$ for $Z \in H_{2}(E)$. Relative to the splitting of $H_{2}(E)$ coming from $p_{1}$ (where the monodromy lies in $\left.\mathcal{I}_{g}\right)$, in particular we must determine $\left[F_{2}\right] \cdot \Sigma_{b, z}$, where $b \in H_{1}\left(B_{1}\right)$ and $z \in H_{1}\left(F_{1}\right)$.

Lemma 7.2 Take $x, y \in H_{1}\left(B_{2}\right)$ satisfying $x \cdot y=1$. For $b \in H_{1}\left(B_{1}\right)$ and $z \in H_{1}\left(F_{1}\right)$, let $\Sigma_{b, z}$ be the associated element of $H_{2}(E)$. Then

(9) $\left[F_{2}\right] \cdot \Sigma_{b, z}=i_{B_{1}}(P x, b) i_{F_{1}}(Q y, z)-i_{B_{1}}(P y, b) i_{F_{1}}(Q x, z)+\tau(b)(Q x \wedge Q y \wedge z)$.

In particular, if $z \in\langle Q x, Q y\rangle^{\perp}$ then (9) simplifies to

$$
\left[F_{2}\right] \cdot \Sigma_{b, z}=\tau(b)(Q x \wedge Q y \wedge z) .
$$

In fact, for all $z \in H_{1}\left(F_{1}\right)$ there exist pairs $x_{z}, y_{z} \in H_{1}\left(B_{2}\right)$ such that $z \in\left\langle Q x_{z}, Q y_{z}\right\rangle^{\perp}$ holds, so that, for all $b$ and $z,(10)$ is satisfied for this choice of $x_{z}$ and $y_{z}$.

Proof The formulas in (9) and (10) follow directly from the description of the intersection form given in Proposition 5.9. The existence of a suitable $x$ and $y$ for a given $z$ is nothing but a matter of symplectic linear algebra. Since we will use some features of the construction later on, we give a detailed explanation. Lemma 7.1 shows that $W=\operatorname{Im} Q$ is a symplectic subspace of $H_{1}\left(F_{1}\right)$, so we can take a symplectic complement $W^{\perp}$. Any $z$ can therefore be written as $w+w^{\prime}$ with $w \in W$ and $w^{\prime} \in W^{\perp}$. If $w=0$ there is nothing to show. Otherwise, extend $w$ to a symplectic basis for $W$ such that $w=x_{1}$. As $B_{2}$ has genus at least 2, this basis includes $x_{2}$ and $y_{2}$ and, as $W=\operatorname{Im} Q$, we can select $x_{z}$ and $y_{z}$ in $H_{1}\left(B_{2}\right)$ with $Q x_{z}=x_{2}$ and $Q y_{z}=y_{2}$.

We conclude this subsection by amalgamating the work we have done in the previous two propositions in order to give a description of $\left[F_{2}\right]$. 
Lemma 7.3 Let $p_{2}: E \rightarrow B_{2}$ be a second fibering. The choice of partial section $\sigma: B^{\prime} \rightarrow E^{\prime}$ furnishes $H_{2}(E)$ with the splitting

$$
H_{2}(E)=\left\langle\left[F_{1}\right]\right\rangle \oplus\left(H_{1}\left(B_{1}\right) \otimes H_{1}\left(F_{1}\right)\right) \oplus H_{2}\left(B_{1}\right),
$$

with $H_{1}\left(B_{1}\right) \otimes H_{1}\left(F_{1}\right)$ spanned by the set of $\Sigma_{b, z}$ where $b$ and $z$ range in symplectic bases $\mathcal{B}$ and $\mathcal{F}$ for $H_{1}\left(B_{1}\right)$ and $H_{1}\left(F_{1}\right)$, respectively, and $H_{2}\left(B_{1}\right)$ is spanned by $C$, as in Proposition 5.9. Relative to this splitting of $\mathrm{H}_{2}(E)$ there is the following expression for $\left[F_{2}\right]$ :

$$
\left[F_{2}\right]=(\delta-2 d e)\left[F_{1}\right]+d C+\sum_{b \in \mathcal{B}, z \in \mathcal{F}} \tilde{\tau}(b)\left(Q x_{z} \wedge Q y_{z} \wedge z\right) \Sigma_{\hat{b} \hat{z}} .
$$

Here, $\delta=i_{B_{1}}(P x, P y)+Q x \cdot Q y \cdot C$ for any choice of $x, y \in H_{1}\left(B_{2}\right)$ satisfying $x \cdot y=1, e=C^{2}$ and $d=\left[F_{1}\right] \cdot\left[F_{2}\right]$ (the algebraic intersection of the two fibers). Also, $\hat{x}$ denotes the symplectic dual of $x$ relative to the chosen symplectic basis.

Proof Suppose $V$ is a free $\mathbb{Z}$-module equipped with a nondegenerate symmetric bilinear pairing $\langle\cdot, \cdot\rangle$. Suppose, moreover, that there exists a generating set $\mathcal{A}=\left\{a_{1}, \ldots, a_{k}, b_{1}, \ldots, b_{k}\right\}$ with the property that $\left\langle a_{i}, a_{j}\right\rangle=\left\langle b_{i}, b_{j}\right\rangle=0$ for all $i$ and $j,\left\langle a_{i}, b_{j}\right\rangle=0$ for $i \neq j$, and $\left\langle a_{i}, b_{i}\right\rangle=1$. Then any element $x \in V$ is expressible in the form

$$
x=\sum_{i=1}^{k}\left\langle x, a_{i}\right\rangle b_{i}+\sum_{i=1}^{k}\left\langle x, b_{i}\right\rangle a_{i} .
$$

We will apply this to $V=H_{2}(E)$ with the intersection pairing; in order to do this we must find a suitable generating set $\mathcal{A}$. Via Proposition 5.9, the space $H_{1}\left(B_{1}\right) \otimes H_{1}\left(F_{1}\right)$ is orthogonal under - to $H_{2}\left(B_{2}\right)$ and to $H_{2}\left(F_{1}\right)$ and, moreover, the collection of $\Sigma_{b, z}$ for $(b, z) \in \mathcal{B} \times \mathcal{F}$ is such a generating set on this subspace. We also have $\left[F_{1}\right] \cdot C=1$ as well as $\left(\left[F_{1}\right]\right)^{2}=0$ and $C^{2}=e$. Therefore, we can take

$$
\mathcal{A}=\left\{\left[F_{1}\right], C-e\left[F_{1}\right]\right\} \cup\left\{\Sigma_{b, z} \mid(b, z) \in \mathcal{B} \times \mathcal{F}\right\} .
$$

The only intersection that remains to be computed is $\left[F_{2}\right] \cdot C$. As $P x \cdot P y=$ $i_{B_{1}}(P x, P y)\left[F_{1}\right]$, a direct computation gives

$$
\begin{aligned}
{\left[F_{2}\right] \cdot C } & =(P x \cdot P y+P x \cdot Q y-P y \cdot Q x+Q x \cdot Q y) \cdot C \\
& =P x \cdot P y \cdot C+Q x \cdot Q y \cdot C \\
& =i_{B_{1}}(P x, P y)+Q x \cdot Q y \cdot C=\delta .
\end{aligned}
$$

By assumption, $\left[F_{1}\right] \cdot\left[F_{2}\right]=d$, and $(10)$ computes $\left[F_{2}\right] \cdot \Sigma_{b, z}$. Therefore we may insert these computations into (12) to obtain (11). 
Rigidity in the Johnson kernel We now assume, as is required for Theorem 1.1, that the monodromy of $p_{1}$ is contained in $\mathcal{K}_{g}$. As noted in the previous section, the closed Johnson kernel $\mathcal{K}_{g}$ coincides with the kernel of $\tau: \mathcal{I}_{g} \rightarrow \wedge^{3} H / H$; similarly, the pointed Johnson kernel $\mathcal{K}_{g, *}$ is the kernel of $\tau: \mathcal{I}_{g, *} \rightarrow \wedge^{3} H$. We also noted above that if $\tau \circ \rho: H_{1}(B) \rightarrow \bigwedge^{3} H / H$ is identically zero then there is a canonical lift $\tilde{\tau}: H_{1}(B) \rightarrow \bigwedge^{3} H$, namely zero. This furnishes the (co)homology of $E$ with a canonical splitting in which all cup products in (10) vanish.

In order to prove the main result of this section, we will compute $\left[F_{2}\right]$ and see that it is "as simple as possible" in the coordinates coming from $p_{1}$, the fibering with monodromy in $\mathcal{K}_{g}$. This will be accomplished via Lemma 7.3. Per our choice of lift $\tilde{\tau}$, the terms expressed via the Johnson homomorphism all vanish, so that

$$
\left[F_{2}\right]=a\left[F_{1}\right]+d C
$$

for some $a \in \mathbb{Z}$. The coefficient $a$ is determined by $\left[F_{2}\right] \cdot C$ or, equivalently, by $\delta=i_{B_{2}}(P x, P y)$ (by Proposition 5.9(3), $Q x \cdot Q y \cdot C=0$ ). This can be determined from Lemma 7.2.

Lemma 7.4 Let $E$ be a 4-manifold with two fiberings as a surface bundle over a surface, $p_{1}: E \rightarrow B_{1}$ and $p_{2}: E \rightarrow B_{2}$. Define the projection $P: H_{1}\left(B_{2}\right) \rightarrow H_{1}\left(B_{1}\right)$. Suppose that the monodromy for the bundle structure associated to $p_{1}$ lies in $\mathcal{K}_{g}$. Then $P \equiv 0$ and, consequently $\delta=0$.

Proof Returning to (9), in the Johnson kernel setting $\left[F_{2}\right] \cdot \Sigma_{b, z}$ and $\tilde{\tau}(b)(Q x \wedge Q y \wedge z)$ are both zero for all $x, y$ and $z$. Taking $z$ to be any element satisfying $i_{F_{1}}(Q y, z) \neq 0$ and $i_{F_{1}}(Q x, z)=0,(9)$ simplifies to $i_{B_{1}}(P x, b)=0$. Since this is true for all $b$, we conclude that $P x=0$ and, since any $x \in H_{1}\left(B_{2}\right)$ has a suitable $y$ such that (9) holds, we conclude that $P \equiv 0$ and $\delta=0$, as claimed.

With this in hand, we can apply Lemma 7.3 (recalling from Proposition 5.9(3) that $e=0$ ) to see that $\left[F_{2}\right]$ is as simple as possible:

$$
\left[F_{2}\right]=d C \text {. }
$$

As was noted following the statement of Proposition 3.1, $\left[F_{2}\right]$ must be a primitive class, so $d= \pm 1$. We conclude that $d=1$ (as $d \geq 0$ by Proposition 4.1 ). We record this fact for later reference:

Lemma 7.5 Let $p_{1}: E \rightarrow B_{1}$ be a surface bundle over a surface with monodromy in $\mathcal{K}_{g}$. Suppose there is a second fibering $p_{2}: E \rightarrow B_{2}$. Then

$$
\operatorname{deg}\left(p_{1} \times p_{2}\right)=1 \text {. }
$$


Proposition 4.1 asserts the equality of $\operatorname{deg}\left(p_{1} \times p_{2}\right)$ with $\operatorname{deg}\left(\left.p_{2}\right|_{F_{1}}: F_{1} \rightarrow B_{2}\right)$ and $\operatorname{deg}\left(\left.p_{1}\right|_{F_{2}}: F_{2} \rightarrow B_{1}\right)$. Consequently,

$$
\operatorname{deg}\left(\left.p_{2}\right|_{F_{1}}: F_{1} \rightarrow B_{2}\right)=\operatorname{deg}\left(\left.p_{1}\right|_{F_{2}}: F_{2} \rightarrow B_{1}\right)=1 .
$$

Remark 7.6 Observe that Lemma 7.5 supplies a proof of the missing assertion $(1) \Longrightarrow$ (3) in Proposition 2.2, namely that, if $E$ is a surface bundle over a surface with monodromy in the Johnson kernel, then any second fibering necessarily yields a bi-projection with nonzero degree. Of course, the assertion that any of the conditions (1)-(3) of Proposition 2.2 are equivalent to the bundle $E$ being a product is the content of Theorem 1.1.

Cohomology: Splittings coming from multisections In order to complete the proof of Theorem 1.1, we will combine the work we have done above with an analysis of what the (co)homology of $E$ looks like with respect to the coordinates coming from the second fibering (where the monodromy need not be contained in $\mathcal{I}_{g}$ ). The most convenient setting for this portion of the argument is in the cohomology ring, so we pause briefly to establish some preliminaries.

Most of what we have established vis-à-vis the intersection pairing on $H_{*}(E)$ is directly portable to the setting of the cup product in cohomology. In particular, the maps

$$
p_{i}^{*}: H^{*}\left(B_{i}\right) \rightarrow H^{*}(E)
$$

for $i=1,2$, are injections. We let $\eta_{i} \in H^{2}\left(B_{i}\right)$ be an integral generator compatible with the chosen orientations; it is easy to see that $p_{i}^{*}\left(\eta_{i}\right)$ is Poincare dual to $\left[F_{i}\right]$. Relative to a choice of splitting

$$
H^{1}(E)=p_{1}^{*} H^{1}\left(B_{1}\right) \oplus H^{1}\left(F_{1}\right),
$$

there are the projection maps $P: H^{1}\left(B_{2}\right) \rightarrow H^{1}\left(B_{1}\right)$ and $Q: H^{1}\left(B_{2}\right) \rightarrow H^{1}\left(F_{1}\right)$, and Lemma 7.4 carries over to show that $P \equiv 0$. We can also transport our analysis of the intersection form on $H_{*}(E)$. In the cohomological setting, we have proved:

Proposition 7.7 Let $F_{1} \rightarrow E \rightarrow B_{1}$ be a surface bundle over a surface with monodromy in the Johnson kernel $\mathcal{K}_{g}$. Then $E$ is an integral cohomology $B_{1} \times F_{1}$, ie there exists a canonical isomorphism

$$
H^{*}(E) \approx H^{*}\left(B_{1}\right) \otimes H^{*}\left(F_{1}\right)
$$

as graded rings.

We now continue with the proof of Theorem 1.1. 
Lemma 7.8 Suppose that the genus of $B_{2}$ is strictly smaller than that of $F_{1}$. Then there exist classes $x, y \in H^{1}(E)$ annihilating $p_{2}^{*} H^{1} B_{2}$ (ie $x \smile p_{2}^{*} z=y \smile p_{2}^{*} z=0$ for all $z \in H^{1}(B)$ ), such that $x \smile y=\Phi_{1}$, where $\Phi_{1} \in H^{2}\left(F_{1}\right)$ is a generator.

Proof The cohomological formulation of Lemma 7.4 shows that

$$
p_{2}^{*} H^{1}\left(B_{2}\right) \leq H^{1}\left(F_{1}\right) .
$$

By (the cohomological reformulation of) Lemma 7.1, $p_{2}^{*} H^{1}\left(B_{2}\right)$ is in fact a symplectic subspace of $H^{1}(F)$, so there exists a symplectic complement. We can then take the desired $x$ and $y$ to be suitable elements of this complement.

To finish the proof of Theorem 1.1, we will examine where $x$ and $y$ must live, relative to coordinates on $H^{*}(E)$ coming from the fibering $p_{2}$. At this point, the results of Section 6 come into play. In particular, $(8)$ endows $H^{1}(E, \mathbb{Q})$ with a splitting

$$
H^{1}(E, \mathbb{Q})=\operatorname{Im} p^{*} \oplus \operatorname{ker} \widehat{\sigma}^{*} .
$$

For the remainder of the proof, we will assume that all of our cohomology groups have rational coefficients.

Lemma 7.9 Let $p: E \rightarrow B$ be any surface bundle over a surface with multisection $\sigma$. Suppose that there exists $x \in H^{1}(E)$ annihilating $p^{*} H^{1}(B)$. Then $x \in \operatorname{ker} \widehat{\sigma}^{*}$.

\section{Proof Write}

$$
x=v+p^{*} b
$$

with $v \in \operatorname{ker} \widehat{\sigma}^{*}$ and $b \in H^{1}(B)$. If $b \neq 0$, then there exists $c \in H^{1}(B)$ with $b \smile c \neq 0$. On the one hand, $x \smile p^{*} c=0$, by assumption. On the other, letting $[B] \in H_{2}(B)$ denote the fundamental class, we have by Proposition 6.4 that

$$
\begin{aligned}
\left\langle x \smile p^{*} c, \hat{\sigma}_{*}[B]\right\rangle & =\left\langle\left(v+p^{*} b\right) \smile p^{*} c, \hat{\sigma}_{*}[B]\right\rangle \\
& =\left\langle v \smile p^{*} c, \hat{\sigma}_{*}[B]\right\rangle+\left\langle p^{*}(b \smile c), \hat{\sigma}_{*}[B]\right\rangle \\
& =0+\left\langle\hat{\sigma}^{*} p^{*}(b \smile c),[B]\right\rangle \\
& =\langle b \smile c,[B]\rangle \neq 0,
\end{aligned}
$$

since $v \in \operatorname{ker} \widehat{\sigma}^{*}$. In this case we have reached a contradiction, so $b=0$ as desired.

Lemma 7.10 Let $F_{1} \rightarrow E \rightarrow B_{1}$ be a surface bundle over a surface with monodromy in $\mathcal{K}_{g}$ and suppose there is a second fibering $p_{2}: E \rightarrow B_{2}$. Let $g$ denote the genus of $F_{1}$ and $h$ denote the genus of $B_{2}$. Then $g=h$. 
Proof We have already established (see Lemma 7.5) that

$$
\operatorname{deg}\left(p_{2} \mid F_{1}\right)=1 \text {. }
$$

As $p_{2}$ has positive degree, we conclude immediately that $g \geq h$. Suppose $g>h$. Then there exist classes $x, y \in H^{1}(E)$ as in the statement of Lemma 7.8. We will make use of the existence of a multisection $\sigma$ of $p_{2}: E \rightarrow B_{2}$ so that, by Lemma 7.9, we must have $x, y \in \operatorname{ker} \hat{\sigma}^{*}$. So, by Proposition 6.4,

$$
\left\langle x \smile y, \hat{\sigma}_{*}\left[B_{2}\right]\right\rangle=0 .
$$

In the notation of Proposition 7.7, both $p_{2}^{*} H^{1}\left(B_{2}\right)$ and the classes $x$ and $y$ are contained in $H^{1}\left(F_{1}\right)$ and, as the image of

$$
\smile: \bigwedge^{2} H^{1}\left(F_{1}\right) \rightarrow H^{2}\left(F_{1}\right)
$$

is one-dimensional (since $F_{1}$ is a surface), we conclude that $x \smile y=p_{2}^{*}\left(\eta_{2}\right)$, where $\eta_{2} \in H_{2}\left(B_{2}\right)$ is a generator. So, then

$$
\left\langle x \smile y, \widehat{\sigma}_{*}\left[B_{2}\right]\right\rangle=\left\langle p_{2}^{*}\left(\eta_{2}\right), \hat{\sigma}^{*}\left[B_{2}\right]\right\rangle=\left\langle\eta_{2},\left[B_{2}\right]\right\rangle=1 .
$$

This is a contradiction; necessarily $g=h$.

This shows that $\left.p_{2}\right|_{F_{1}}$ is a map of degree one between surfaces of the same genus and thus, as is well known,

$$
\left(p_{2}\right)_{*}: \pi_{1} F_{1} \rightarrow \pi_{1} B_{2}
$$

must be an isomorphism.

End of proof of Theorem 1.1 At this point, we turn to an analysis of the fundamental group. Via the long exact sequence in homotopy for a fibration, there is an exact sequence

$$
1 \rightarrow \pi_{1} F_{i} \rightarrow \pi_{1} E \rightarrow \pi_{1} B_{i} \rightarrow 1
$$

for $i=1,2$. Consequently, the kernel of

$$
\left(p_{1} \times p_{2}\right)_{*}: \pi_{1} E \rightarrow \pi_{1} B_{1} \times \pi_{1} B_{2}
$$

is given by $\pi_{1} F_{1} \cap \pi_{1} F_{2}$. On the other hand, this is also the kernel of the crossprojection

$$
\pi_{1} F_{1} \rightarrow \pi_{1} B_{2}
$$

which was just shown to be an isomorphism. We conclude that $\left(p_{1} \times p_{2}\right)_{*}$ is an isomorphism. 
The monodromy of the bundle $E$ can be read off from the fundamental group as the map $\pi_{1} B_{1} \rightarrow \operatorname{Out}\left(\pi_{1} F_{1}\right) \approx \operatorname{Mod}\left(\Sigma_{g}\right)$ (the latter isomorphism coming from the theorem of Dehn, Nielsen, and Baer). Since $\pi_{1} E$ is a product, this map is trivial. The correspondence (1) then shows that $E$, being a surface bundle with trivial monodromy, is diffeomorphic to $B_{1} \times B_{2}$. This completes the proof of Theorem 1.1.

\section{References}

[1] MF Atiyah, The signature of fibre-bundles, from: "Global analysis (papers in honor of K Kodaira)", (D C Spencer, S Iyanaga, editors), Univ. Tokyo Press (1969) 73-84 MR0254864

[2] A Cavicchioli, F Hegenbarth, D Repovš, On four-manifolds fibering over surfaces, Tsukuba J. Math. 22 (1998) 333-342 MR1650733

[3] B Farb, D Margalit, A primer on mapping class groups, Princeton Mathematical Series 49, Princeton Univ. Press (2012) MR2850125

[4] V Guillemin, A Pollack, Differential topology, Prentice-Hall, Englewood Cliffs, NJ (1974) MR0348781

[5] U Hamenstädt, On surface subgroups of mapping class groups, talk at the workshop "Hot topics: Surface subgroups and cube complexes", MSRI (2013) Available at https://www.msri.org/workshops/723/schedules/16639

[6] A Hatcher, Algebraic topology, Cambridge Univ. Press (2002) MR1867354

[7] J A Hillman, Four-manifolds, geometries and knots, Geometry \& Topology Monographs 5 (2002) MR1943724

[8] F E A Johnson, A rigidity theorem for group extensions, Arch. Math. (Basel) 73 (1999) 81-89 MR1703674

[9] K Kodaira, A certain type of irregular algebraic surfaces, J. Analyse Math. 19 (1967) 207-215 MR0216521

[10] S Morita, Characteristic classes of surface bundles, Invent. Math. 90 (1987) 551-577 MR914849

[11] S Morita, Geometry of characteristic classes, Translations of Mathematical Monographs 199, Amer. Math. Soc. (2001) MR1826571

[12] I Rivin, Rigidity of fibering, preprint (2011) arXiv:1106.4595

[13] I Rivin, Statistics of 3-manifolds occsionally fibering over the circle, preprint (2014) arXiv: 1401.5736

[14] N Salter, Surface bundles over surfaces with arbitrarily many fiberings, Geom. Topol. 19 (2015) 2901-2923 MR3416116 
Department of Mathematics, University of Chicago

5734 S University Ave, Chicago, IL 60637, USA

nks@math . uchicago . edu

http://math.uchicago.edu/ nks/

Received: 7 December $2014 \quad$ Revised: 29 March 2015 